\title{
Dielectric Spectroscopy of Polymer-Based Nanocomposite Dielectrics with Tailored Interfaces and Structured Spatial Distribution of Fillers
}

\author{
G. Polizos, E. Tuncer, V. Tomer, I. Sauers, C.A. Randall, and E. Manias
}

\section{CONTENTS}

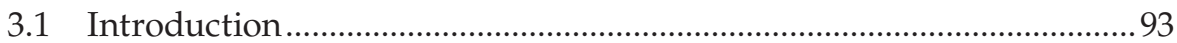

3.2 Broadband Dielectric Relaxation Spectroscopy .....................................95

3.2.1 Dielectric Relaxations................................................................ 95

3.2.2 Dielectric Formalisms ............................................................97

3.2.2.1 Permittivity Formalism ............................................97

3.2.2.2 Electric Modulus Formalism .......................................98

3.2.2.3 Impedance Formalism.................................................99

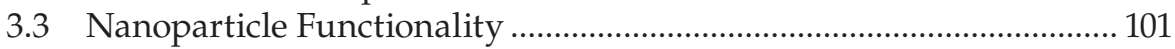

3.3.1 Segmental Dynamics and Conductivity Mechanism ............. 101

3.3.2 Interfacial Dynamics ............................................................... 106

3.3.3 In Situ Particle Nucleation ........................................................ 113

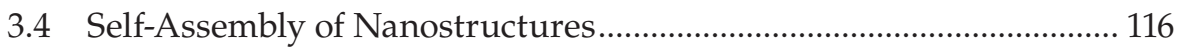

3.5 Hierarchical Structures .................................................................... 120

3.5.1 Thermoset-Based Nanocomposites ........................................ 120

3.5.2 Thermoplastic-Based Nanocomposites ................................... 122

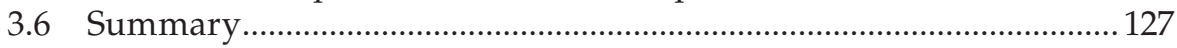

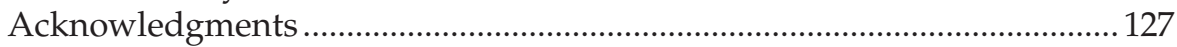

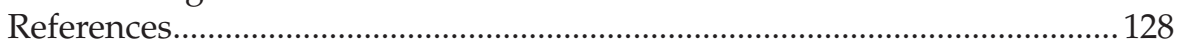

\subsection{Introduction}

Emerging technologies in the areas of electronics and energy storage require the design of next-generation dielectric-component materials with welldefined structure and properties with higher performance under voltage and temperature. Polymers filled with inorganic nanoparticles are potential 
candidates for breakthrough advances, since they enable the integration of constituents with desirable properties, particularly when hierarchically structuring the filler phase. However, implementation of highly innovative material elements necessitates the fundamental understanding of each element's properties and interactions at the nanoscale and, further, how those are manifested in the macroscopic performance of the nanocomposites. Dielectric spectroscopy, coupled with performance measurements, is crucial in quantifying such properties and unveiling the molecular-level interdependencies and their correlations with device performances.

The chapter summarizes recent studies on the nanostructure-property interdependencies in tailored/structured polymer nanocomposites, and underlines the nanoscale principles leading to the design and synthesis of high-performance nanomaterials for energy storage applications. In principle, inorganic materials of high permittivity combined with polymers of high breakdown strength should improve the energy storage capacity, as energy density is directly proportional to permittivity and the square of the highest applied electric field. This is not always the case, and real composites rarely exhibit a behavior that is a straight-forward addition of filler and matrix response. The main drawbacks in such composite approaches most often relate to the polymer-filler interfaces, in particular, when these interfaces are characterized by properties adverse to the desired macroscopic behavior. With increasing filler surface area, and depending on the polymerfiller interactions, the presence of such interfaces can govern the macroscopic properties of the composite and also determine properties such as the dielectric breakdown strength. The research work in this chapter provides clues on the mitigation of such detrimental effects, which are commonly responsible for the poor dielectric performance of the composites. Three key factors are emphasized for synthesizing multifunctional nanocomposites:

1. Nanoparticle functionality: The uniform and controlled dispersion of the inorganic fillers is a challenge in the fabrication of nanocomposites. The fundamental thermodynamics of mixing for polymers and nanofillers are described through a balance of entropic and enthalpic factors. Two fabrication routes are presented: (a) functionalization of the particle surface in order to tailor the polymer/particle interfacial interactions and (b) in situ nucleation of nanoparticles in a polymer solution. The polymer dynamics (relaxations) in the vicinity of the nanoscale boundaries are investigated by means of dielectric relaxation spectroscopy (DRS). Of particular interest is when the size of the particles $(\sim 5 \mathrm{~nm})$ is comparable to the length-scale of the polymer conformations.

2. Self-assembly of desired nanostructures: The mismatch in the permittivity and conductivity between the organic and the inorganic phases generates concentration in the local electric field that may lead to premature electrical failure of the nanodielectric. Self-assembled structures of fillers with different dielectric properties can be employed 
to grade the local electric field and, thus, increase the energy density of the composites. We functionalized and covalently bonded two different inorganic constituents to yield a combined filler that has a thermodynamically favorable dispersion and a capability to covalently bond to the polymer matrix. In this section, we summarize our results on the dynamical behavior of the polymer phase, including those polymers adsorbed on filler surfaces or confined in between fillers. The dynamics of the polymer phase relaxing at the inorganic interfaces is correlated with the macroscopic polarization of the composites under nonlinear electric fields.

3. Hierarchical nanostructures: Nanocomposites with controlled spatial distribution of fillers are, arguably, the most promising route to optimize macroscopic response. Such nanocomposites, with fillers structured appropriately over the various length scales, can be synthesized using anisotropic dielectrophoretic assembly or flow/stress-gradient induced alignment techniques. The orientation of the fillers is quantitatively analyzed in terms of Hermans orientation factors by employing two-dimensional x-ray diffraction measurements. We emphasize on the important role of conductivity, complex permittivity, and, particularly, local cluster distribution and relative orientation in controlling high-field dielectric behavior. It is shown that filler alignment can be used to markedly improve the high electric-field breakdown strength and, consequently, the recoverable energy density.

A wide range of materials are investigated to gain insights into the generally applicable mechanisms that control the dielectric breakdown strength and the nonlinear conduction, namely, thermoset (epoxy, polydimethylsiloxane) and thermoplastic (polyethylene, polyurethane) polymers are studied, reinforced by model inorganic nanofillers such as barium titanate, titanium dioxide, and montmorillonite. In the following sections, we present a brief introduction to the dielectric spectroscopy and analysis tools. The remainder of the chapter focuses on the fundamental interrelationships between the nanoscale dielectric properties and the macroscopic properties of the nanocomposites with an emphasis on the dielectric breakdown strength.

\subsection{Broadband Dielectric Relaxation Spectroscopy}

\subsubsection{Dielectric Relaxations}

DRS is a dynamic technique quantifying the molecular dynamics and conductivity processes in dielectric (insulating or semiconducting) materials due to their interaction with electromagnetic fields. In a linear system, 
when an external ac electric field is applied to a dielectric medium with permanent dipole moments, the complex dielectric function $\varepsilon^{*}(\omega)=\varepsilon^{\prime}(\omega)$ $i \varepsilon^{\prime \prime}(\omega)$ is associated with the correlation function $\Phi(t)$ of the polarization fluctuations [1-3]

$$
\frac{\varepsilon^{*}(\omega)-\varepsilon_{\infty}}{\varepsilon_{s}-\varepsilon_{\infty}}=\int_{0}^{\infty} \frac{-d \Phi(t)}{d t} \exp (-i \omega t)
$$

where

$$
\Phi(t)=\frac{\langle\Delta P(t) \Delta P(0)\rangle}{\left\langle\Delta P(0)^{2}\right\rangle}
$$

The angular brackets denote a statistical ensemble average of the fluctuation of the polarization $P$ around its equilibrium value; $\omega(=2 \pi f)$ stands for the angular frequency and $\varepsilon_{s}, \varepsilon_{\infty}$ are the low- and high-frequency limits of the real part of permittivity. Dielectric measurements can be performed either in the frequency domain (i.e., the sample is excited/polarized by an external sinusoidal electric field and the complex impedance, $Z(\omega)$, is measured) or in the time domain (i.e., the sample is subjected to a stepfunction voltage and the dielectric functions are derived from the reflection and transmission coefficients). As an example, the correlation function $\Phi(t)$ can describe the decay of electric polarization when an external electric field is removed from a dielectric material. For a single relaxation time $\tau$ (i.e., identical non-interacting dipoles), $\Phi(t)$ is an exponential of the form $\exp (-t / \tau)$ and the dielectric dispersion (Debye) is obtained from Equation 3.1

$$
\frac{\varepsilon^{*}(\omega)-\varepsilon_{\infty}}{\varepsilon_{s}-\varepsilon_{\infty}}=\frac{1}{1+i \omega \tau}
$$

where the real and imaginary parts are, respectively,

$$
\begin{aligned}
& \varepsilon^{\prime}(\omega)=\varepsilon_{\infty}+\frac{\varepsilon_{s}-\varepsilon_{\infty}}{1+\omega^{2} \tau^{2}} \\
& \varepsilon^{\prime \prime}(\omega)=\frac{\varepsilon_{s}-\varepsilon_{\infty}}{1+\omega^{2} \tau^{2}} \omega \tau
\end{aligned}
$$

The correlation function for materials with dipole groups of different reorientation polarization will decay in multiple steps giving rise to several relaxation processes. Moreover, heterogeneous systems or systems with strongly interacting dipoles, such as polymers and polymer composites, are 
described by the Kohlrausch-Williams-Watts (KWW) function (stretched exponential)

$$
\Phi(t)=\exp \left[-\left(\frac{t}{\tau_{K W W}}\right)^{\beta_{K W W}}\right], \quad 0<\beta_{K W W} \leq 1
$$

that leads to broad and asymmetric distribution of relaxation times. Analysis of the dielectric dispersions can quantify the dynamic parameters associated with the material's structure in the molecular level and specifically (1) the dielectric relaxation strength $\left(\Delta \varepsilon=\varepsilon_{s}-\varepsilon_{\infty}\right)$, which is a measure of the orientation polarization and can be expressed in terms of the dipole number density and mean square dipole moment; (2) the time scale and activation energy of relaxations arising either from the rotational motion of polar chain segments or from cooperative reorientations associated with the glass transition; (3) the distribution of relaxation times and their correlation with intra- and intermolecular interactions; (4) the ac and dc conductivity values, activation energy, and length-scale of the translational diffusion of mobile ions.

DRS supplements other dynamic techniques such as mechanical spectroscopy, nuclear magnetic resonance, neutron and light scattering; however, the main advantage of dielectric spectroscopy is its ability to follow relaxation processes over an extremely wide range of characteristic times $\left(10^{5}-10^{-12} \mathrm{~s}\right)$. The following sections present the fundamental concepts of the mathematical formalisms and analysis tools used herein for the interpretations of our experimental results.

\subsubsection{Dielectric Formalisms}

\subsubsection{Permittivity Formalism}

A superposition of Havriliak-Negami (HN) expressions [1,4-7] was employed for the analysis of the complex permittivity function

$$
\varepsilon^{*}(\omega)=\varepsilon_{\infty}+\sum_{i=1}^{n} \frac{\Delta \varepsilon_{i}}{\left[1+\left(i \omega \tau_{0, i}\right)^{1-\alpha_{i}}\right]^{\beta_{i}}}
$$

Least-square fitting to the experimental data was carried out by sharing the common parameters $\Delta \varepsilon_{i}, \tau_{0, i}, \alpha_{i}, \beta_{i}$ in the real

$$
\varepsilon^{\prime \prime}(\omega)=\sum_{i=1}^{n} \Delta \varepsilon_{i} \frac{\cos \left(\beta_{i} \phi_{i}\right)}{\left[1+2\left(\omega \tau_{0, i}\right)^{1-\alpha_{i}} \sin \left(\frac{1}{2} \alpha_{i} \pi\right)+\left(\omega \tau_{0, i}\right)^{2\left(1-\alpha_{i}\right)}\right]^{\beta_{i} / 2}}
$$


and imaginary part

$$
\varepsilon^{\prime \prime}(\omega)=\sum_{i=1}^{n} \Delta \varepsilon_{i} \frac{\sin \left(\beta_{i} \phi_{i}\right)}{\left[1+2\left(\omega \tau_{0, i}\right)^{1-\alpha_{i}} \sin \left(\frac{1}{2} \alpha_{i} \pi\right)+\left(\omega \tau_{0, i}\right)^{2\left(1-\alpha_{i}\right)}\right]^{\beta_{i} / 2}}
$$

of the complex permittivity function. A conductivity power law term $\left(\sigma_{0} / \varepsilon_{0}\right)$ $\omega^{-s}$ was added in the imaginary part (Equation 3.9) when contribution from conductivity was present in the datasets. In the earlier equations $\alpha, \beta$ are the shape parameters defining the symmetrical and asymmetrical broadening of each mode $(0<(1-\alpha) \beta, s \leq 1)$. The argument $(\beta \phi)$ denotes the angle from $\varepsilon_{\infty}$ to any value of the $\varepsilon^{*}(\omega)$ function in a complex locus diagram [5,8] $\left(\varepsilon^{\prime \prime}(\omega)\right.$ vs. $\left.\varepsilon^{\prime}(\omega)\right)$ where

$$
\varphi_{i}=\arctan \left[\frac{\left(\omega \tau_{o, i}\right)^{1-\alpha_{i}} \cos \left(\alpha_{i} \pi / 2\right)}{1+\left(\omega \tau_{o, i}\right)^{1-\alpha_{i}} \sin \left(\alpha_{i} \pi / 2\right)}\right]
$$

and $\tau_{o}$ is a characteristic time that is associated to the relaxation time $\tau_{\max }\left(=1 / 2 \pi f_{\max }\right)$ of each mode by

$$
\tau_{\max , i}=\tau_{o, i}\left[\frac{\sin \left[\left(1-a_{i}\right) \beta_{i} \pi /\left(2+2 \beta_{i}\right)\right]}{\sin \left[\left(1-a_{i}\right) \pi /\left(2+2 \beta_{i}\right)\right]}\right]^{1 /\left(1-\alpha_{i}\right)}
$$

\subsubsection{Electric Modulus Formalism}

The electric modulus was introduced by McCrum et al. [2] and is defined as the reciprocal complex permittivity [9-11]

$$
M^{*}(\omega)=\frac{1}{\varepsilon^{*}(\omega)}=M^{\prime}(\omega)+i M^{\prime \prime}(\omega)=\frac{\varepsilon^{\prime}(\omega)}{\varepsilon^{\prime 2}(\omega)+\varepsilon^{\prime \prime 2}(\omega)}+i \frac{\varepsilon^{\prime \prime}(\omega)}{\varepsilon^{\prime 2}(\omega)+\varepsilon^{\prime \prime 2}(\omega)}
$$

It is widely used to suppress conductivity and polarization phenomena and to reveal the dipolar contributions. Macedo et al. [12-14] applied this formalism to ionic conductors. The authors approached the dielectric response by an equivalent electric circuit of a parallel capacitance and conductance element configuration. The Debye type relaxation time that corresponds to this circuit (or equivalently to the diffusion of the ions in the material) is expressed as the conductivity relaxation time

$$
\tau_{\sigma}=\frac{\varepsilon_{o} \varepsilon_{s}}{\sigma_{d c}}, \quad \mathbf{E}=\mathbf{E}_{\mathbf{o}} \varphi(t)=\mathbf{E}_{\mathbf{o}} \exp \left(\frac{-t}{\tau_{\sigma}}\right)
$$


and describes the decay of the electric field, $\mathrm{E}$, when the electric displacement vector, $\mathrm{D}$, remains constant. In a more general case, the single relaxation time (Debye) model can be extended to a distribution of relaxation times [12].

The complex electric modulus in the frequency domain can be written as the Fourier transform of the time derivative of the electric field decay function,

$$
M^{*}(\omega)=M_{\infty}\left[1-\int_{0}^{\infty}-\frac{d \varphi(t)}{d t} \exp (-i \omega t) d t\right]
$$

In the frequency domain, a corresponding to the $\mathrm{HN}$ expression can be obtained in the modulus level. Tsangaris et al. $[15,16]$ resolved the imaginary and real part for different distributions of relaxation times. A HN distribution will reduce to

$$
M^{\prime}(\omega)=M_{\infty} M_{s} \frac{\left[M_{s} A^{\beta}+\left(M_{\infty}-M_{s}\right) \cos \beta \varphi\right] A^{\beta}}{M_{s}^{2} A^{2 \beta}+2 A^{\beta}\left(M_{\infty}-M_{s}\right) M_{s} \cos \beta \varphi+\left(M_{\infty}-M_{s}\right)^{2}}
$$

and

$$
M^{\prime \prime}(\omega)=M_{\infty} M_{s} \frac{\left[\left(M_{\infty}-M_{s}\right) \sin \beta \varphi\right] A^{\beta}}{M_{s}^{2} A^{2 \beta}+2 A^{\beta}\left(M_{\infty}-M_{s}\right) M_{s} \cos \beta \varphi+\left(M_{\infty}-M_{s}\right)^{2}}
$$

where

$$
\begin{aligned}
& M_{s}=1 / \varepsilon_{s} \\
& M_{\infty}=1 / \varepsilon_{\infty}
\end{aligned}
$$

and

$$
A=\left[1+2\left(\omega \tau_{o}\right)^{1-\alpha} \sin \left(\frac{\alpha \pi}{2}\right)+\left(\omega \tau_{o}\right)^{2(1-\alpha)}\right]^{1 / 2}
$$

The argument $\varphi$ and the characteristic time $\tau_{0}$ have similar dependencies as those in Equations 3.10 and 3.11.

\subsubsection{Impedance Formalism}

The conductivity processes in multiphase systems can be identified and separated in terms of the complex impedance formalism, $Z^{*}(\omega)=Z^{\prime}(\omega)-i Z^{\prime \prime}(\omega)$, where the measured real and imaginary parts are connected with the complex permittivity and the geometric capacity of the free space $\left(C_{0}\right)$

$$
Z_{\text {meas. }}^{\prime}(\omega)=\varepsilon^{\prime \prime}(\omega)\left(\left[\varepsilon^{\prime 2}(\omega)+\varepsilon^{\prime \prime 2}(\omega)\right] \omega C_{0}\right)^{-1}
$$




$$
Z_{\text {meas. }}^{\prime \prime}(\omega)=\varepsilon^{\prime}(\omega)\left(\left[\varepsilon^{2}(\omega)+\varepsilon^{\prime \prime 2}(\omega)\right] \omega C_{0}\right)^{-1}
$$

A typical data representation is based on plots of the imaginary versus the real part, also known as Nyquist plots. In such plots, the overall measured complex impedance can be expressed as the superposition of the intrinsic impedance (bulk contribution described by semicircles) and a dispersive capacitance (constant phase element, CPE) contribution describing electrode polarization effects at low frequencies [17].

$$
Z_{\text {meas. }}^{*}(\omega)=Z_{\text {intrinsic }}^{*}(\omega)+A_{C P E}(i \omega)^{-s}
$$

In the previous expression, the values of the exponent $s$ vary between 0 and 1 for ideal resistive and capacitive behavior, respectively. The real and imaginary components of the intrinsic impedance can be fitted with analytical expressions similar to Equations 3.8 and 3.9

$$
\begin{aligned}
& Z_{\text {intrinsic }}^{\prime \prime}(\omega)=R_{\infty}+\sum_{i=1}^{n} \frac{\Delta R_{i} \cos \left(\beta_{i} \phi_{i}\right)}{\left[1+2\left(\omega \tau_{0, i}\right)^{1-\alpha_{i}} \sin \left(\frac{1}{2} \alpha_{i} \pi\right)+\left(\omega \tau_{0, i}\right)^{2\left(1-\alpha_{i}\right)}\right]^{\beta_{i} / 2}} \\
& Z_{\text {intrinsic }}^{\prime \prime}(\omega)=\sum_{i=1}^{n} \frac{\Delta R_{i} \sin \left(\beta_{i} \phi_{i}\right)}{\left[1+2\left(\omega \tau_{0, i}\right)^{1-\alpha_{i}} \sin \left(\frac{1}{2} \alpha_{i} \pi\right)+\left(\omega \tau_{0, i}\right)^{2\left(1-\alpha_{i}\right)}\right]^{\beta_{i} / 2}}
\end{aligned}
$$

where

$R_{\infty}$ is the resistance at the high-frequency limit

$\tau_{0, i}$ and $\Delta R_{i}$ are the characteristic time and the resistance values for each impedance mode, respectively

The $\Delta R_{i}$ values correspond to the intersection of the extrapolated to lowfrequencies semicircle and the real $Z^{\prime}$ axis. Conductivity $\sigma$ is obtained by normalizing $\Delta R$ values to the geometric parameters according to

$$
\sigma=(\Delta R)^{-1} \frac{d}{S}
$$

where

$d$ is the thickness

$S$ is the surface of the sample

Phenomenological models based on equivalent circuits can simulate the polarization mechanisms and the dissipative contributions due to mobile charges. 


\subsection{Nanoparticle Functionality}

\subsubsection{Segmental Dynamics and Conductivity Mechanism}

Nanoparticle-filled polymers (nanodielectrics) have gained remarkable scientific attention because of their potential use in a variety of applications ranging from polymer electrolytes to electrical insulation [18-22]. In this section, we report on the correlation between the cooperative segmental dynamics and the conductivity mechanism. Presynthesized titanium dioxide $\left(\mathrm{TiO}_{2}\right)$ nanoparticles functionalized with polyethylene glycol (PEG) were dispersed in a thermoplastic polyurethane (PU) with polar soft segments [23]. The particles were approximately $10 \mathrm{~nm}$ in size and composites were obtained at low-weight fractions $(0.42$ and $0.10 \mathrm{wt} \%)$ to avoid the formation of pronounced aggregates.

The dynamical properties of the unfilled PU were investigated by means of DRS. Three relaxations are shown in Figure 3.1 for temperatures below room temperature. In the order of increasing temperature, the first two subglass modes, $\gamma$ and $\beta$, are associated with local motions of chain segments. Specifically, the $\gamma$ process is associated with the rotation of polar groups due to the crankshaft motion of $\left(\mathrm{CH}_{2}\right)_{n}$ sequences, whereas the $\beta$ process is due to the orientation fluctuations of ester complexes

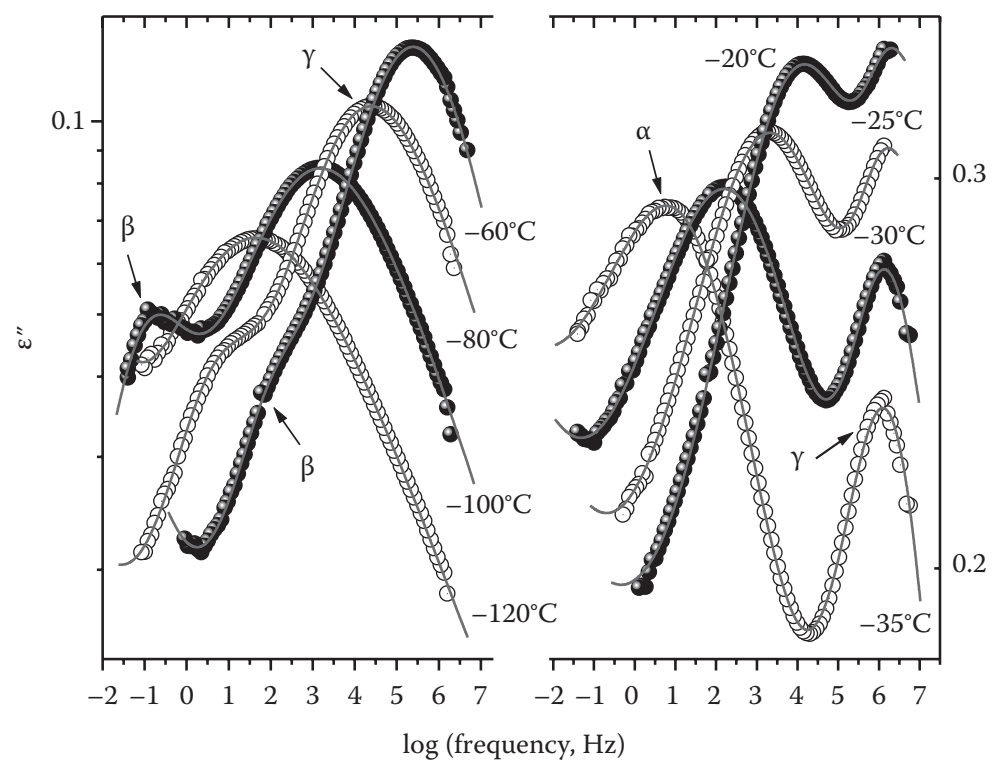

FIGURE 3.1

Imaginary part of permittivity at several temperatures for the unfilled polyurethane matrix. The cooperative $\alpha$ (associated with the glass transition) and the local $\beta, \gamma$ relaxations are indicated on the plot. The lines are the best fits of Equation 3.9 to the data. 
$-\left(\mathrm{CH}_{2}\right)_{4}-\mathrm{O}(\mathrm{C}=\mathrm{O}) \mathrm{N}-$ with participation of attached water molecules [24-29]. The segmental $\alpha$ relaxation at higher temperatures is positioned close to the calorimetric glass transition temperature, $T_{g}$, and is attributed to dipole moment cooperative reorientations, during the glass transition of the soft segment microdomains. In the composites, this process is overlapping with a low-frequency conductivity contribution due to variations in the surface charge density of the $\mathrm{TiO}_{2}$ [23].

Significant changes have been observed in the segmental dynamics of the composites and a comparison plot is shown in Figure 3.2. At high temperatures, the composites exhibit the same dynamical behavior as the pristine PU. However, upon temperature decreasing toward the glass transition temperature, the segmental $\alpha$ mode is found to depend on the particle content;

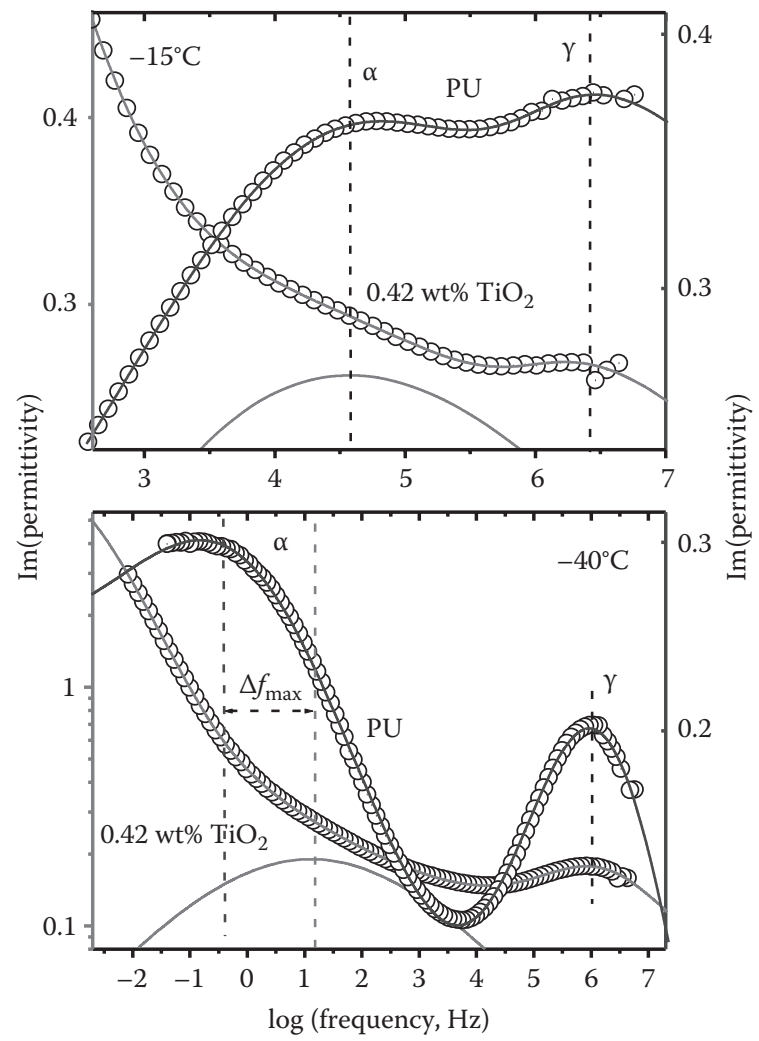

FIGURE 3.2

Comparison plot of the dielectric relaxations for the pristine polyurethane and the $0.42 \mathrm{wt} \%$ in $\mathrm{TiO}_{2}$. No shift is observed in the peak maximum frequencies of the local $\gamma$ relaxations. However, upon temperature decreasing toward the glass transition temperature, the segmental $\alpha$ mode of the composite shifts 1.5 decades toward higher frequencies and becomes faster compared to the $\alpha$ relaxation in the pristine polyurethane. (From Polizos, G. et al., Polymer, $53,595,2012$.) 


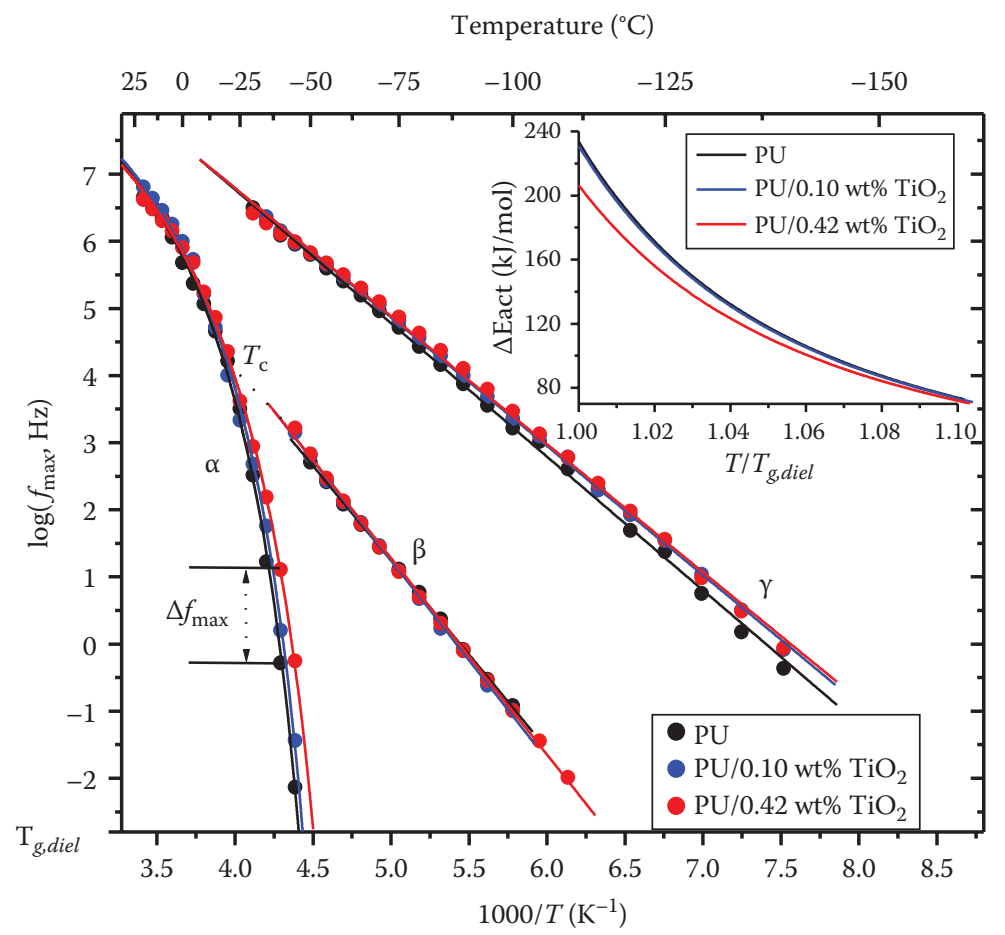

FIGURE 3.3

(See color insert.) Summarized Arrhenius plot of the cooperative $(\alpha)$ and local $(\beta, \gamma)$ relaxations at temperatures below room temperature along with the fitting lines according to Equations 3.24 and 3.25. $T_{c}$ corresponds to the merging temperature of the $\alpha$ and $\beta$ relaxations. $\Delta f_{\max }$ is the frequency shift of the $\alpha$ relaxation (for the $0.42 \mathrm{wt} \%$ composite at $-40^{\circ} \mathrm{C}$ ) according to Figure 3.2. The dielectric glass transition $T_{g, \text { diel }}$, obtained at $1.6 \mathrm{mHz}(\tau=100 \mathrm{~s})$, is also indicated on the plot. The normalized (by $T_{8}$ ) apparent activation energies that correspond to the $\alpha$ relaxations were calculated from Equation 3.27 and are shown in the inset plot. (From Polizos, G. et al., Polymer, 53, 595, 2012.)

and specifically for the $0.42 \mathrm{wt} \%$ composite, it shifts 1.5 decades toward higher frequencies and becomes faster compared to the $\alpha$ relaxation in the unfilled PU. The time scale dependence for all the relaxations is shown in Figure 3.3. The temperature dependence of $f_{\max }$ for the subglass modes, $\gamma$ and $\beta$, is described by the Arrhenius equation

$$
f_{\max , \beta, \gamma}(T)=f_{\infty} \exp \left(\frac{-\Delta E_{A}}{R T}\right)
$$

where

$\Delta E_{A}$ is the activation energy of the process

$f_{\infty}$ is the relaxation rate in the high-temperature limit

$R$ is the universal gas constant 


\section{TABLE 3.1}

Summarized Fitting Analysis Results according to Equations 3.24 and 3.25 for the Frequency Dependence of the Relaxations in Figure 3.3

\begin{tabular}{lcccccccc}
\hline & \multicolumn{5}{c}{$\boldsymbol{\alpha}$ Relaxation } & & \multicolumn{2}{c}{$\boldsymbol{\beta}, \boldsymbol{\gamma}$ Relaxations } \\
\cline { 2 - 4 } Sample & ${ }^{a} \boldsymbol{T}_{\boldsymbol{g}}^{\text {DSC }}(\mathbf{C})$ & ${ }^{b} \boldsymbol{T}_{\boldsymbol{g}}^{\text {diel }}(\mathbf{C})$ & ${ }^{c} \boldsymbol{T}_{\mathbf{0}}(\mathbf{C})$ & ${ }^{d} \boldsymbol{m}$ & ${ }^{e} \boldsymbol{D}$ & & ${ }^{f} \boldsymbol{\Delta} \boldsymbol{E}_{\boldsymbol{\beta}}(\mathbf{k J} / \mathbf{m o l})$ & ${ }^{g} \Delta \boldsymbol{E}_{\boldsymbol{\gamma}}(\mathbf{k J} / \mathbf{m o l})$ \\
\hline PU & -43.6 & -46.1 & -69.8 & 124 & 3.5 & & $54.0 \pm 0.3$ & $38.1 \pm 0.3$ \\
$0.10 \mathrm{wt} \%$ & -45.3 & -47.5 & -71.2 & 123 & 3.5 & & $57.0 \pm 0.6$ & $36.8 \pm 0.2$ \\
$0.42 \mathrm{wt} \%$ & -48.3 & -50.9 & -76.7 & 112 & 3.9 & & $56.0 \pm 0.6$ & $36.5 \pm 0.3$ \\
\hline
\end{tabular}

Source: Reproduced from Polizos, G. et al., Polymer, 53, 595, 2012.

${ }^{a} T_{8}^{D S C}$ is the calorimetric glass transition temperature according to differential scanning calorimetry thermograms (not shown here).

${ }^{b} T_{g}^{\text {diel }}$ is the dielectric glass transition temperature obtained from the extrapolation of Equation 3.7 to $1.6 \mathrm{mHz}(\tau=100 \mathrm{~s})$.

${ }^{c} T_{0}$ is the Vogel fitting parameter in Equation 3.25.

$d_{m}$ is the fragility index calculated from Equation 3.26.

${ }^{e} D$ is the strength parameter that is proportional to the inverse fragility.

${ }^{f} \Delta E_{\beta}$ is the activation energy of the $\beta$ relaxation obtained from Equation 3.24.

${ }^{g} \Delta E_{\gamma}$ is the activation energy of the $\gamma$ relaxation obtained from Equation 3.24.

The respective activation energies were calculated from Equation 3.24 and are summarized in Table 3.1. The local dynamics and the activation energies in the pristine PU and in the composites were found to be similar, indicating that the local conformations are independent of the PU-particle interactions.

The time scale of the $\alpha$ relaxation in Figure 3.3 can be described by the Vogel-Tammann-Fulcher (VTF) equation

$$
f_{\text {max }, \alpha}(T)=A \exp \left[\frac{-B}{\left(T-T_{0}\right)}\right]
$$

where $A, B$, and $T_{0}$ (Vogel temperature) are temperature-independent empirical constants listed in Table 3.1. The ideal glass transition temperature, $T_{0}$, has been found for glass-forming polymers to be approximately 40-50 K lower than the $T_{g}$, and it is defined as the temperature where the configurational entropy of the undercooled liquid is zero. In that respect, $T_{0}$ values correlate rather well with the calorimetric and dielectric glass transition temperatures $\left(T_{g}^{D S C}\right.$ and $T_{g}^{\text {diel }}$ in Table 3.1). The variation in the $T_{g}$ values is inherently related to the distinct dynamics of the $\alpha$ relaxation, previously discussed in Figure 3.2. The fragility $(m)$ can be used to quantify this deviation $[30,31]$

$$
m=\left.\frac{d \log \langle\tau\rangle}{d\left(T_{g} / T\right)}\right|_{T=T_{g}}=B T_{g}\left(T_{g}-T_{0}\right)^{-2} \log e
$$

where $m$ is a dimensionless steepness index and is defined as the slope of the relaxation times $(\tau=1 / 2 \pi f)$ versus the inverse temperature at the glass 
transition temperature. The fragility is associated with the inter- and intramolecular interactions, and index $m$ is a measure of the bond interactions during the vitrification of a glass-forming liquid (i.e., the extreme values are $m=16$ for strong Arrhenius-like glass-forming liquids with directional bonds, and $m=250$ for fragile polymers with nondirectional bonds [32-34]). The fragility values, derived from the VTF fitting parameters, as well as the strength parameter $(D)$, which is proportional to the inverse fragility $\left(D=B / T_{0}\right)$, are summarized in Table 3.1. A significant decrease in the fragility of the polymer matrix is observed for the $0.42 \mathrm{wt} \%$ nanocomposite. Typically, the fragility depends on the particle-polymer interactions and is increasing or decreasing for attractive and repulsive interactions, respectively [35-37]. In spite of the hydrogen bond formation between the $\mathrm{TiO}_{2}$ particles and the PU matrix [23], both the $T_{g}$ and the fragility were found to decrease. The favorable formation of hydrogen bonds between the functionalized $\mathrm{TiO}_{2}$ fillers and the hard segments of the PU [23] hinder the cross-linking between the soft and the hard segments and results to soft segment domains with faster segmental dynamics that change more gradually than the dynamics of the corresponding domains in the unfilled PU during the glass formation. It should be noted that the decrease of the fragility index is indirectly related to the $\mathrm{TiO}_{2}$ nanoparticles. Typically, variations in the fragility of nanocomposites are due to changes in the geometrical frustration and polymer packing density. Herein, the observed changes originate from the weaker interactions between the hard and the soft segments, which also lead to a decrease in the glass transition temperature. This is better illustrated in the inset plot (Figure 3.3), showing the normalized (by $T_{g}$ ) temperature dependence of the apparent activation energy $\left(\Delta E_{a c t}^{*}\right)$ for the $\alpha$ relaxation. It is calculated from the derivative of Equation 3.25 and is expressed as a function of the VTF parameters and the universal gas constant $(R)[38]$

$$
\frac{\Delta E_{a c t}^{*}}{R}=\frac{d \log f_{\max }}{d(1 / T)}=B T^{2}\left(T-T_{0}\right)^{-2} \log e
$$

The apparent activation energy of the $0.42 \%$ composite is decreased approximately $11 \%$ when the temperature is equal to the glass transition temperature due to the weaker interactions between the hard and the soft segments. On the contrary, when the temperature is higher than the critical $\alpha-\beta$ merging temperature $\left(T_{c}\right)$, all samples exhibit identical barriers related to the cooperative segmental motion. Since no noticeable changes occur in the local dynamics, this variation in the activation energy is probably associated with fluctuations in the dynamic heterogeneity and cooperativity due to the addition of the nanoparticles $[39,40]$.

The mobility of the polar groups is intrinsically connected to the conduction process, and conductivity was found to dominate the dielectric spectra for temperatures above the critical merging temperature $T_{c}$. The complex 
impedance formalism was employed to analyze the dielectric response of the samples and the respective Nyquist plots are shown in Figure 3.4 for selected temperatures. The pristine PU matrix is characterized by a single mode due to the relaxation of polar groups, whereas a bimodal relaxation is shown in the impedance response of the composites.

Based on the cluster morphology of the composites [23], we may assume a shell model configuration in which the individual $\mathrm{TiO}_{2}$ particles of a cluster are surrounded by a shell-interfacial polymer phase. In this case, the high-frequency relaxation in the composites can be attributed to the intracluster mobility of the ions and specifically to a short length scale motion involving subdiffusion of ions between neighboring particles within a cluster. This motion takes place through the polar segments of the polymer shell, and is characterized by a resistance $\Delta R_{\text {intracluster }}$ In view of the previous discussions, the low frequency-relaxation can be assigned to an intercluster diffusion of the ions. At longer times, the ions will diffuse through the bulk polymer phase and migrate to neighboring clusters. This process is characterized by a resistance $\Delta R_{\text {intercluster }}$ and the overall (bulk) resistance of the composite is the sum of the intra- and intercluster resistance as indicated on the plot. At temperatures greater than $50^{\circ} \mathrm{C}$, the bulk conductivity in the composites

$$
\sigma_{\text {bulk }}=\left(\Delta R_{\text {intracluster }}+\Delta R_{\text {intercluster }}\right)^{-1} \frac{d}{S}
$$

is approximately 2.5 orders of magnitude higher than the conductivity of the unfilled matrix [23].

On the contrary, for temperatures below $T_{g}$, the nanocomposites exhibit significant lower dielectric losses (therefore and conductivity) compared to the pristine PU. The tan $\delta$ values in Figure 3.5 are approximately 1 order of magnitude lower than those in the PU at $-263^{\circ} \mathrm{C}$. This variant dependence of the conductivity mechanism can be utilized in polymer electrolytes as well as in the design of electronic devices operating at broad temperature windows. Similar behavior has been observed in thermoset-based nanocomposites, which is the subject of the following sections.

\subsubsection{Interfacial Dynamics}

The formation of interfaces due to the incorporation of nanoparticles into a polymer host may determine the macroscopic properties of the nanodielectric and result to significant enhancements or detrimental effects depending on the size of the interfaces and their interactions with the polymer matrix. Dielectric spectroscopy is a powerful tool for detecting and analyzing the dynamics of polar segments that relax at the vicinity of nanoparticles even when the particles are at small volume fractions. In this section, we present our recent studies on the interfacial dynamics of epoxy resin composites 


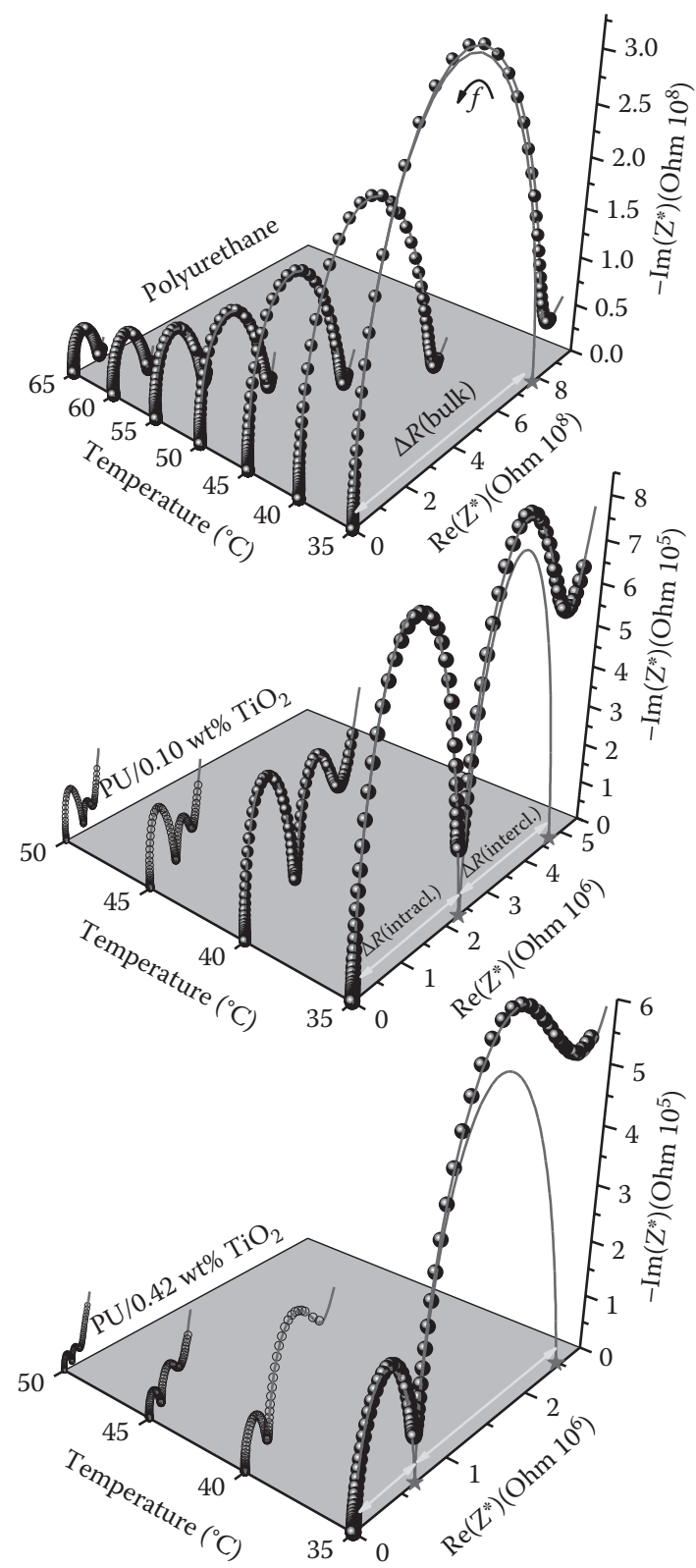

FIGURE 3.4

(See color insert.) Complex impedance Nyquist plots for the unfilled polyurethane and the nanocomposites for several temperatures. The fitting curves to the data are according to Equation 3.20. The conductivity process in the unfilled matrix is described by a single relaxation whereas the nanocomposites are characterized by a bimodal relaxation due to the intraand intercluster conduction processes. (The top two plots are reproduced from Polizos, G. et al., Polymer, 53, 595, 2012.) 


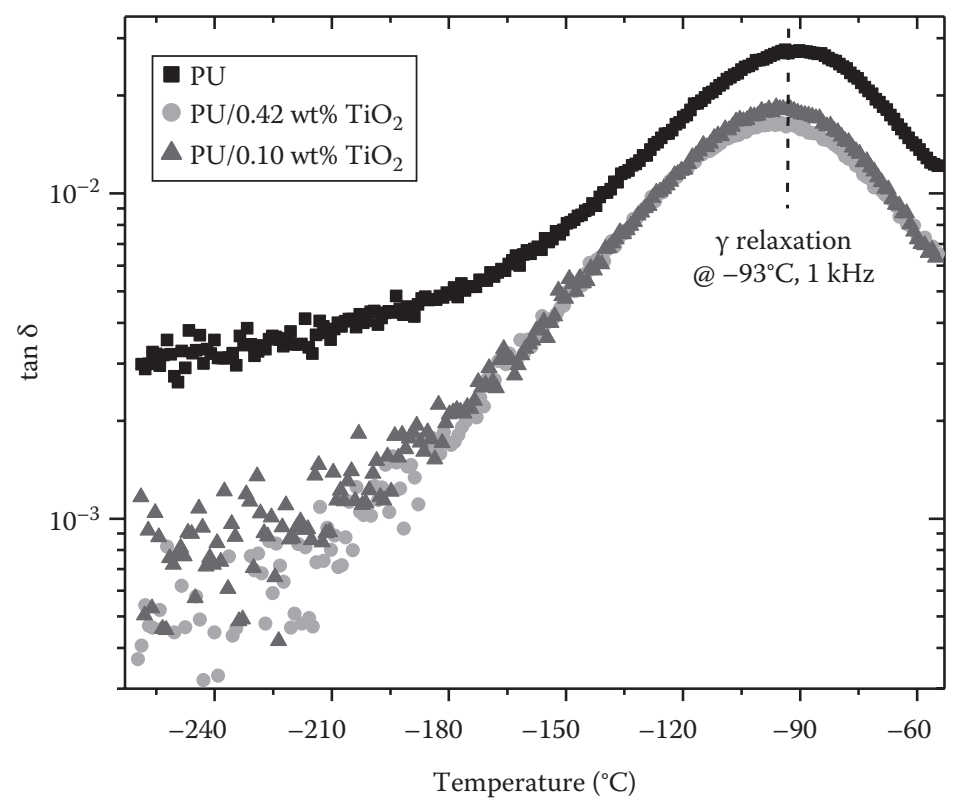

FIGURE 3.5

Isochronal plot for the dielectric tan $\delta$ at $1 \mathrm{kHz}$. The measurements were carried out on a homemade setup, and the $\gamma$ relaxation is in excellent agreement with the measurement results in Figures 3.1 and 3.3. In the low-temperature limit, the composites exhibit significant lower losses compared to the unfilled polyurethane.

based on organically modified montmorillonite (OMMT) and barium titanate (BT). The nanofillers were selected to exploit the high aspect ratio of the montmorillonite (MMT) and the high permittivity of the BT particles, which improve the mechanical performance and the operating electric field of the composites, respectively. Hybrid organic/inorganic composites of two and three phases were prepared by dispersing BT or/and OMMT particles in the epoxy resin [41]. The interfacial dynamics below the glass transition temperature were found to depend on the particle functionalization and weight fraction.

In the temperature range between $T_{g}$ and $40^{\circ} \mathrm{C}$, a new relaxation mode was evident in the composites with 3 and $6 \mathrm{wt} \%$ OMMT, $30 \mathrm{vol} \%$ BT as well as in the three-phase system with both BT and OMMT fillers. This mode was absent in the pristine cross-linked epoxy matrix and in the $1 \mathrm{wt} \%$ OMMT and $10 \mathrm{vol} \%$ BT composites (a comparison plot at $90^{\circ} \mathrm{C}$ is shown in Figure 3.6). Since the dynamics of this mode are dependent on the particle type and weight fraction, we may associate its origin to the formation of interfaces and specifically to the polar segments relaxing at the vicinity of the inorganic fillers. In good agreement with the IR results [41] that show the formation of an uncross-linked polymer phase that becomes pronounced with weight fraction increasing, we can assign the nature of this relaxation to the interfacial 

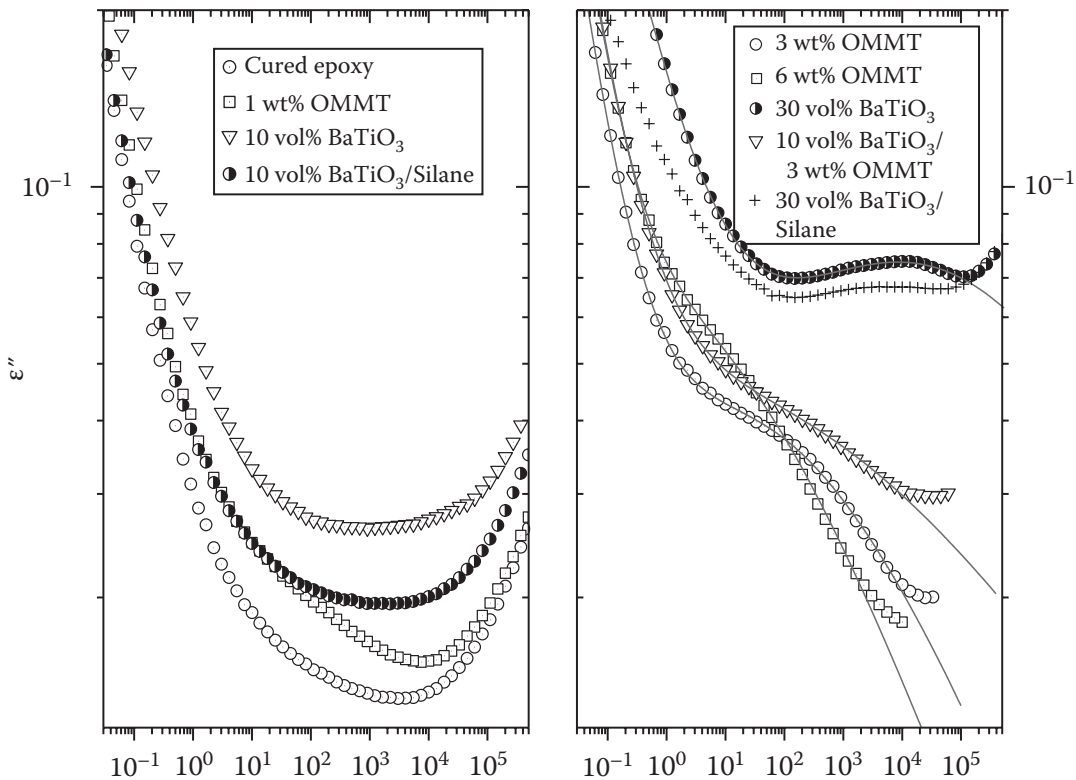

(a)

(b)

Frequency $(\mathrm{Hz})$

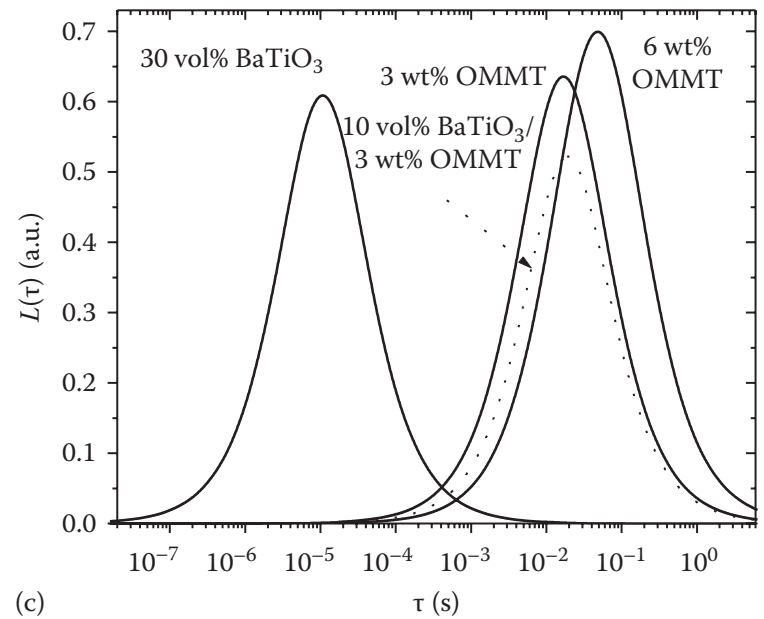

FIGURE 3.6

(a) Summarized dielectric loss plot at $90^{\circ} \mathrm{C}$ for the pristine epoxy matrix and the two- and three-phase composites. (b) A relaxation process is evident in the composites with high filler contents. The Cole-Cole dielectric function was found to give the best fitting to the data and the corresponding distributions of relaxation times are presented in plot (c). (Reproduced from Tomer, V. et al., J. Appl. Phys., 108, 074116, 2010.) 
unreacted (mobile) epoxy monomers. This argument is further supported by the surface modification of the BT, which was found to improve the interfacial properties. Despite the high inorganic content of the $30 \mathrm{vol} \%$ BT composite, the dielectric relaxation strength of the interfacial process is suppressed and becomes negligible when the BT surface is modified with organic coupling agents based on silane functional monolayers (Figure 3.6b). The proper functionality of the fillers allows their integration with the polymer matrix and prevents the formation of mobile interfaces.

To separate the dipolar from the conductivity contributions and therefore better illustrate the dielectric response of the composites, the distributions of relaxation times [1,2], $L(\tau)$, are also presented in Figure 3.6c. This interpretation assumes that the broadening of a relaxation is due to the superposition of Debye processes

$$
\varepsilon^{\prime \prime}(\omega)=\int_{-\infty}^{\infty} \frac{\omega \tau L(\tau)}{1+(\omega \tau)^{2}} d \ln (\tau)
$$

$L(\tau)$ can be calculated from the fitting parameters of the imaginary and real parts of the permittivity function and is analytically written as

$$
L(\tau)=\frac{1}{2 \pi} \frac{\sin (\pi-\alpha \pi)}{\cosh \left(\ln \left(\tau / \tau_{0}\right)\right)+\sin (\pi-\alpha \pi)}
$$

In the same figure, it is clearly shown that the OMMT-based composites are characterized by approximately 3 orders of magnitude slower dynamics compared to the BT composites (shift of the distribution toward higher relaxation times). This shift is the result of the stronger interactions between the MMT surface and the interfacial polymer layer. The latter is geometrically restricted (physisorbed) and is less mobile than in the BT-based composites. The relative position of the interfacial distributions is a measure of the interfacial strength. Additional information can be derived from the shape of the distribution (i.e., the symmetric Cole-Cole distribution in Figure 3.6c is suggesting polymer segments relaxing in homogeneous environments that possibly result from the good dispersion of the fillers). The particle dispersion and the morphology of the particle clusters may also affect the interfacial dynamics. In Figure 3.6c is shown that the interfacial dynamics in the $6 \mathrm{wt} \%$ OMMT composite is slower than in the $3 \mathrm{wt} \%$ composite, despite the fact that both composites are based on the same type of fillers. To understand this behavior, we can assume that the measured interfacial relaxation is the average dielectric response. The hydroxyl groups, formed during the epoxide ring conversion, are possible to hydrogen bond to the silicate surface. Therefore, the observed retardation in the dynamics of the $6 \mathrm{wt} \%$ may reflect an average increase in the population of the hydroxyl 


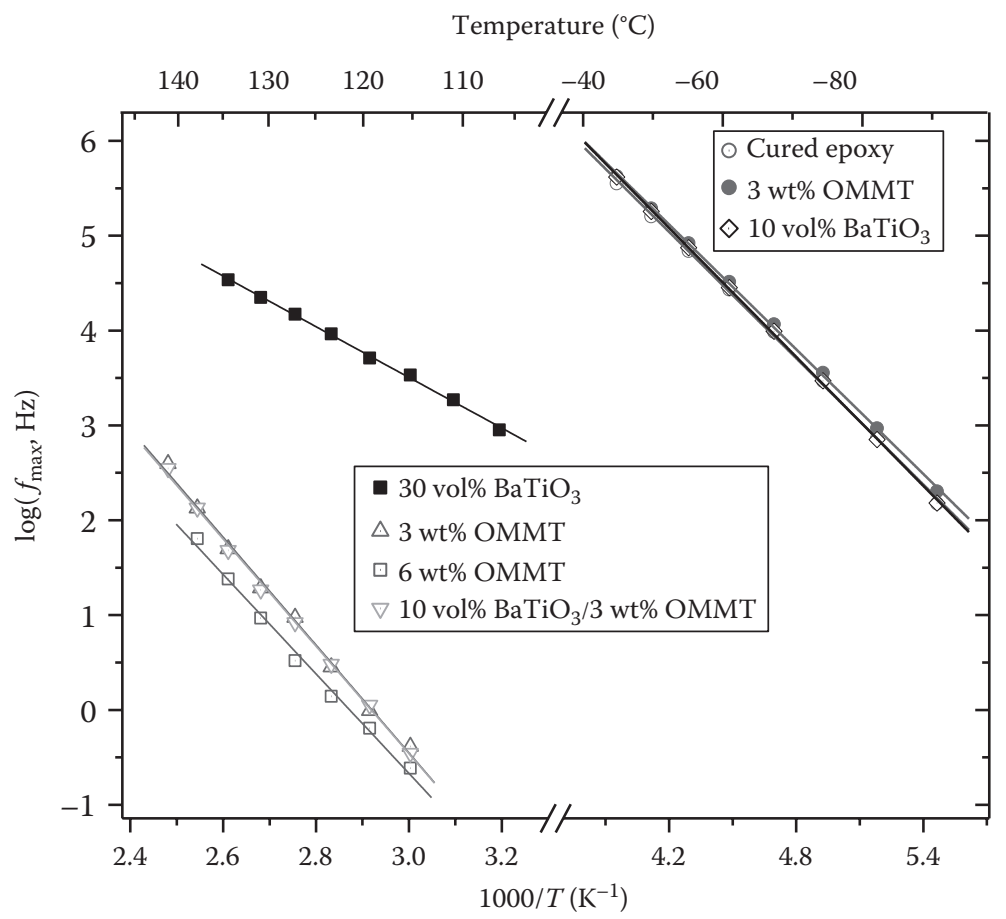

FIGURE 3.7

Summarized Arrhenius plot of the relaxations in selected composites and in the pristine epoxy network. The ultra-fast modes in the subzero temperature range were found to overlap and only three representative compositions are given. A distinct behavior is shown in the interfacial dynamics of the OMMT- and BT-based composites. (Reproduced from Tomer, V. et al., J. Appl. Phys., 108, 074116, 2010.)

groups being attached to the silicate surface, due to a possible increase of the cluster's surface area.

The processes in Figure 3.6b as well as those in the subzero temperature range [41] were analyzed and the summarized Arrhenius plot is presented in Figure 3.7. The subzero modes exhibit dynamics that are several orders of magnitude faster (ultra-fast) compared to those arising from the interfaces. They were found to overlap in all specimens and therefore are independent of the filler type and weight fraction. The respective activation energy for the ultra-fast mode was calculated from Equation 3.24 and was found to be $42 \mathrm{~kJ} / \mathrm{mol}$. This value is in good agreement with previous studies that have identified this relaxation to local reorientations of the hydroxyl groups in the cross-linked epoxy network [42].

The interfacial modes were not only characterized by distinct dynamics depending on the filler type but were also found to exhibit different activation energies. The $\Delta E_{A}$ value for the OMMT-based composites is approximately $105 \mathrm{~kJ} / \mathrm{mol}$, whereas for the $30 \mathrm{vol} \%$ BT composite is $51 \mathrm{~kJ} / \mathrm{mol}$. 
This noteworthy decrease in the energy barriers and the corresponding speed up of the dynamics (previously discussed) are a clear evidence of the weaker interfacial strength between the BT fillers and the epoxy network.

Heterogeneities in the permittivity and conductivity properties between the different constituents in a multiphase composite restrict the mobility of the ionic species (space charge) at the boundaries of different phases. The resulting space-charge interfacial dynamics [43-45] are inherently related to the bulk conductivity of the composite [41]. An example is shown in Figure 3.8 where a relaxation process is evident in the ac conductivity, $\sigma_{a c}(f)$. In this region, conductivity is frequency-dependent and describes a short-range motion of the space-charge carriers (i.e., a subdiffusive type of transport due to sublinear dependence of the mean square displacement on time $[46,47])$. For such short time scales, the charges are confined at heterogeneous boundaries. The thermally activated hopping process of the charges bound at the interfaces gives rise to relaxation phenomena that

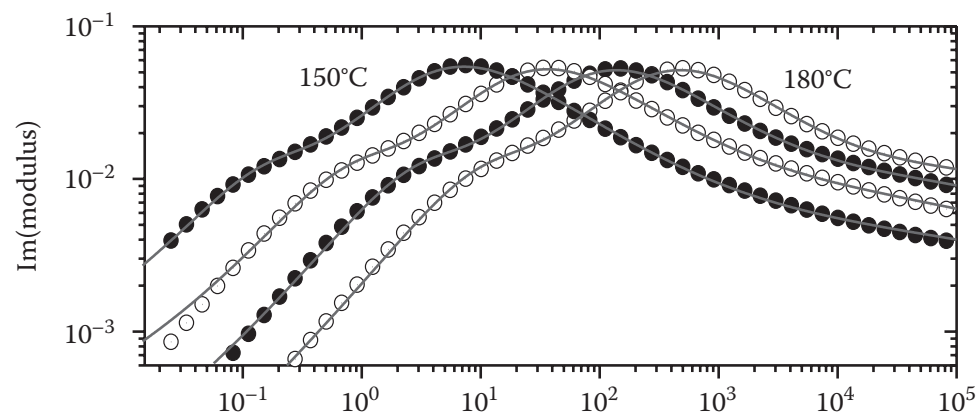

(a)

Frequency $(\mathrm{Hz})$

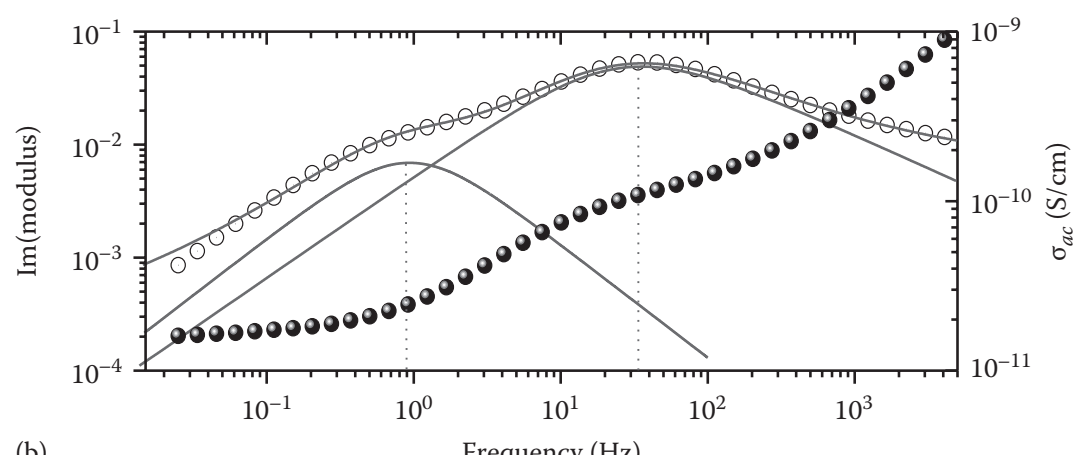

(b)

Frequency $(\mathrm{Hz})$

FIGURE 3.8

(a) The imaginary part of electric modulus for the $10 \mathrm{vol} \% \mathrm{BaTiO}_{3}$ composite at representative temperatures in steps of $10^{\circ} \mathrm{C}$. The lines are the best fits of Equation 3.16 to the experimental data. (b) A comparison plot showing the correspondence between the electric modulus and the ac conductivity at $160^{\circ} \mathrm{C}$. The low-frequency process in the electric modulus corresponds to the onset of dc conductivity, whereas the high-frequency process corresponds to the space-charge interfacial relaxation. (Reproduced from Tomer, V. et al., J. Appl. Phys., 108, 074116, 2010.) 
at longer time scales (lower frequencies) are followed by a diffusive type motion (i.e., dc conductivity; longer times give the ability to the charges to escape from the interfaces and diffuse in the bulk). This is clearly shown in Figure 3.8 where the low-frequency relaxation is in good agreement with the onset of the dc conductivity. In addition to the impedance formalism analysis (see Section 3.1), the electric modulus formalism can also be utilized to separate the conductivity contributions. The dynamics of the different length scale processes depend on the nanoparticle size and functionality and can be correlated with the recoverable energy density of the nanodielectric [41]. To eliminate or suppress the interfacial polarization effects, we present in the following two sections nanocomposites fabricated by in situ and self-assembly techniques.

\subsubsection{In Situ Particle Nucleation}

Titanium dioxide $\left(\mathrm{TiO}_{2}\right)$ nanoparticles, smaller than $5 \mathrm{~nm}$ in diameter, were in situ nucleated in epoxy resin by the hydrolysis and oxidation of $\mathrm{TiCl}_{3}[48-53]$

$$
\begin{gathered}
\mathrm{TiCl}_{3}+3 \mathrm{H}_{2} \mathrm{O} \rightarrow \mathrm{Ti}(\mathrm{OH})_{3}+3 \mathrm{HCl} \\
2 \mathrm{Ti}(\mathrm{OH})_{3}+\frac{1}{2} \mathrm{O}_{2} \rightarrow 2 \mathrm{TiO}_{2}+3 \mathrm{H}_{2} \mathrm{O}
\end{gathered}
$$

The particle size, which is comparable to the length scale of the polymer entanglements, is anticipated to restrict the mobility of the polymer chains [54]. This is clearly shown in the dielectric losses at low temperatures in Figure 3.9. A composite with similar content in $\mathrm{TiO}_{2}$ nanoparticles that were ex situ synthesized and later dispersed in the epoxy is also included for comparison. The absence of pronounced interfaces and particle agglomerations in the in situ composite [54] resulted in dielectric losses that are approximately 1 order of magnitude lower than those in the unfilled epoxy.

The restricted mobility of the polymer chains was found to improve the dielectric breakdown strength of the in situ composite and potentially increase the energy density that can be stored in the nanodielectric. The failure probability distributions of the samples are presented in Figure 3.10. A standard two parameter Weibull model was employed for the data analysis $[55,56]$

$$
P\left(\alpha_{W}, \beta_{W}, E_{B D}\right)=1-\exp \left[-\left(\frac{E_{B D}}{\alpha_{W}}\right)^{\beta_{W}}\right]
$$

In the previous expression, $P$ is the cumulative probability function; $E_{B D}$ is the dielectric breakdown field; and the parameters $\alpha_{W}$ and $\beta_{W}$ denote the 


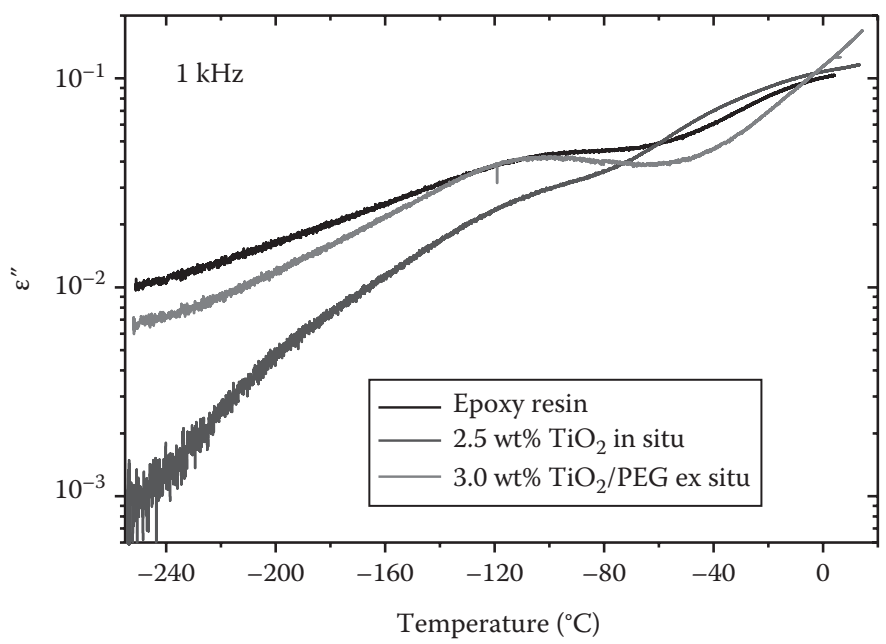

FIGURE 3.9

Dielectric losses at $1 \mathrm{kHz}$ for the pristine epoxy and nanocomposites with similar contents in $\mathrm{TiO}_{2}$ prepared with in situ and ex situ techniques. A significant decrease of the dielectric losses is shown for the in situ composite.
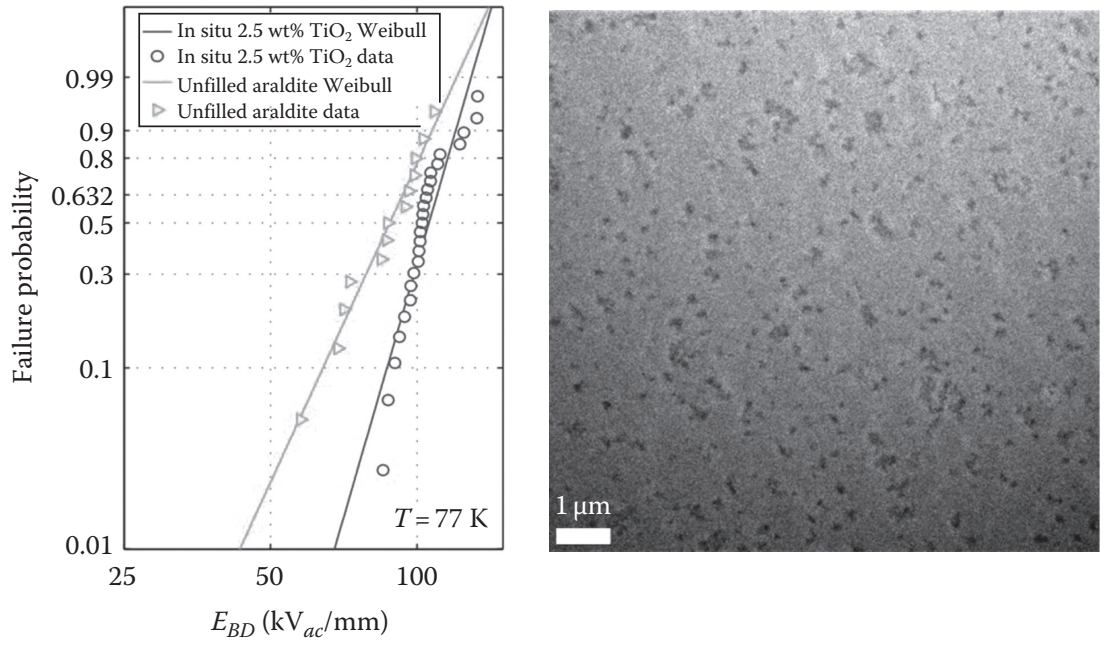

\section{FIGURE 3.10}

Dielectric breakdown failure probability analysis based on Weibull statistics. The samples were submerged in liquid nitrogen and the frequency of the applied ac electric field was $60 \mathrm{~Hz}$. The TEM image corresponds to the in situ $2.5 \mathrm{wt} \%$ composite. (Reproduced from Polizos, G. et al., Appl. Phys. Lett., 96, 152903, 2010.) 
characteristic breakdown strength when the failure probability is 0.6321 and the scattering in the breakdown values, respectively. The improvement in the reliability of the $2.5 \mathrm{wt} \%$ in situ composite ( $\beta_{W}$ values) is approximately $60 \%$ whereas the corresponding benefit in the breakdown strength is $20 \%$ at the characteristic Weibull probability and $56 \%$ at $1 \%$ failure probability. Considering the small weight fraction of the $\mathrm{TiO}_{2}$ phase, the associated improvements are remarkable and extremely significant in the design of high-voltage systems.

The small size and uniform dispersion of the fillers decreased the dielectric losses and narrowed the distribution of relaxation times (narrower peaks in Figure 3.9). However, no shift in the position of the peaks, compared to the unfilled matrix, was observed for the in situ composite. This is also evident in the mechanical relaxations over a broad temperature range, shown in Figure 3.11. The $\alpha$ and the local $\beta, \gamma, \delta$ relaxations are activated in identical temperature windows for all samples, indicating that the nanoparticles did not change the cross-linking density of the polymer matrix and resulted in composites with thermodynamical properties similar to those in the pristine epoxy. Moreover, a 30\% increase in the storage modulus of the $2.5 \mathrm{wt} \%$ composite was observed at the lowest measured temperature.

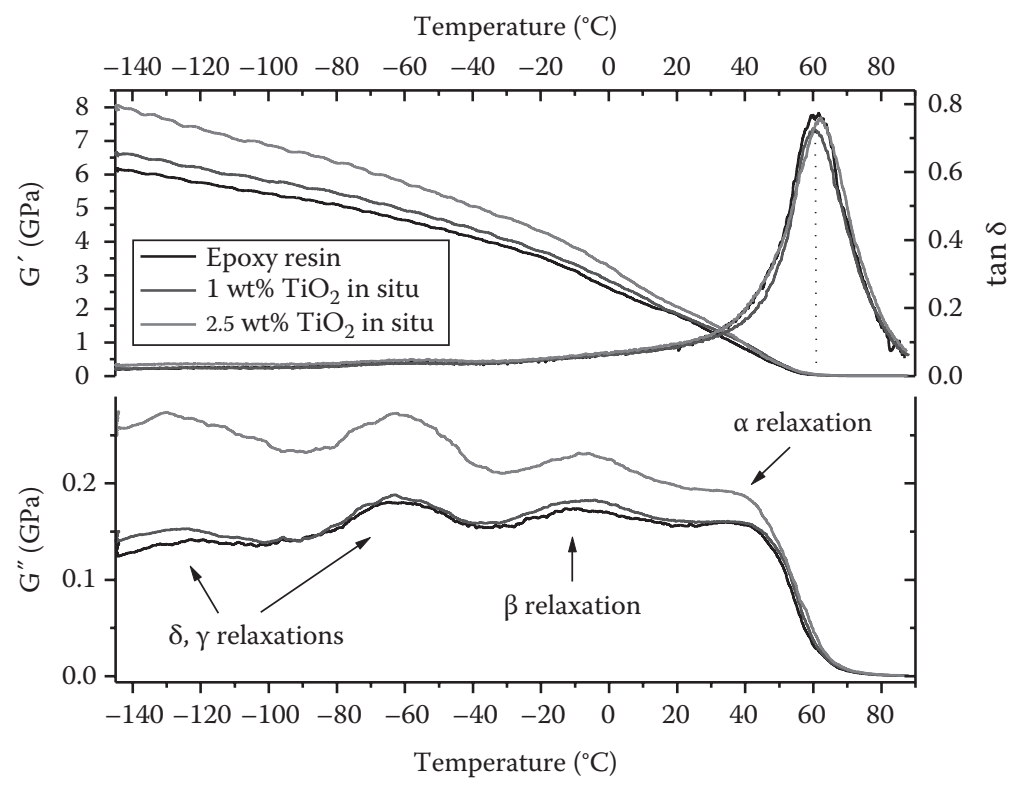

FIGURE 3.11

Storage modulus $\left(G^{\prime}\right)$, loss modulus $\left(G^{\prime \prime}\right)$, and $\tan \delta$ according to dynamic mechanical analysis at $1 \mathrm{~Hz}$. The nanoparticles were in situ synthesized in the polymer matrix. The $\alpha$ relaxation is associated with the glass transition and the $\tan \delta$ peak corresponds to $T_{g}$. 


\subsection{Self-Assembly of Nanostructures}

Nanoparticle functionality and nanoparticles with controlled size have shown notable benefits against detrimental effects associated with the interfaces; however, certain applications may necessitate the integration of two or more inorganic phases. Particles functionalized with reactive agents to form self-assembled nanostructures can strengthen the interfaces and provide vital enhancements in the dielectric properties of the composites.

Interfacial polarization effects predominantly occur due to the mismatch in the permittivity and conductivity values between the organic and the inorganic phases, and typically generate concentration in the local electric field, which may lead to electrical failure of the nanodielectric. Self-assembly nanostructures of fillers with different dielectric properties are possible to grade the local electric field and increase the operating field and therefore the stored electrical energy in the composite (the stored energy is a function of the permittivity and the square of the operating electric field). In view of the earlier discussion, BT and OMMT nanoparticles were covalently bonded and the integrated two-phase hybrid nanofillers yield a thermodynamically favorable dispersion of the particles in the epoxy resin [57]. The morphology of the resultant structures is shown in Figure 3.12. Assembled layered silicates are attached on the surface of the spherical BT particles. The high organic content ( $25 \mathrm{wt} \%)$ of the silicates and their ability to cross-link with the epoxy matrix improved the filler dispersion and created robust interfacial environments that resemble those in the unfilled epoxy. This is evident in the polarization curves (Figure 3.12e) where the high field losses are similar for the pristine epoxy and the composite with reactive fillers. The intrinsic high permittivity of the BT particles increased the high field permittivity (higher slope of the polarization loop) and the recoverable energy density of the composite. Additional improvements related to the mechanical performance $(68 \%$ increase in the storage modulus) and the glass transition temperature were also found [57].

Dielectric spectroscopy can provide insights on the correlation between the properties of the composites and their interfacial dynamics. In Figure 3.13a is shown a comparison plot for temperatures below the glass transition temperature. A relaxation process is present only in the composite with the highest filler content $(20 \mathrm{wt} \%)$. Since the relaxation strength $(\Delta \varepsilon)$ is proportional to the number density of the relaxing units, this process can be ascribed to the formation of a mobile polymer phase that is loosely bound to the inorganic surfaces. At lower particle contents where the particle agglomeration is less pronounced, the interfacial polymer phase is less mobile (more tightly bound on the particle surface [58]) and the corresponding dielectric process is absent or negligible. The strength and dynamics of this process are in good agreement 
with the thermo-mechanical properties of the composites [57] (i.e., heat capacity, onset and width of the glass transition, cross-linking density, and mechanical modulus) and can be used as a measure of the polymer-filler interfacial strength. The dynamics for the $20 \mathrm{wt} \%$ composite in Figure $3.12 \mathrm{~b}$ were obtained from the best fit analysis of the experimental data with a superposition of Equation 3.9 and a conductivity contribution. The time scale dependence of the relaxation process over the entire measured temperature range is shown in Figure 3.13c and the calculated activation energy was found to be $226 \mathrm{~kJ} / \mathrm{mol}$. This process is formed at considerably

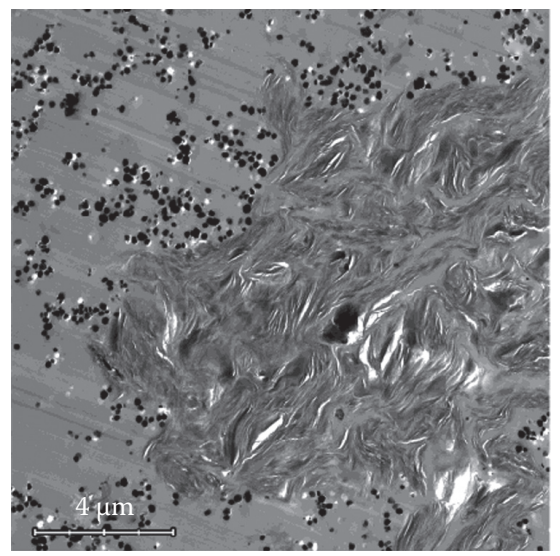

(a)

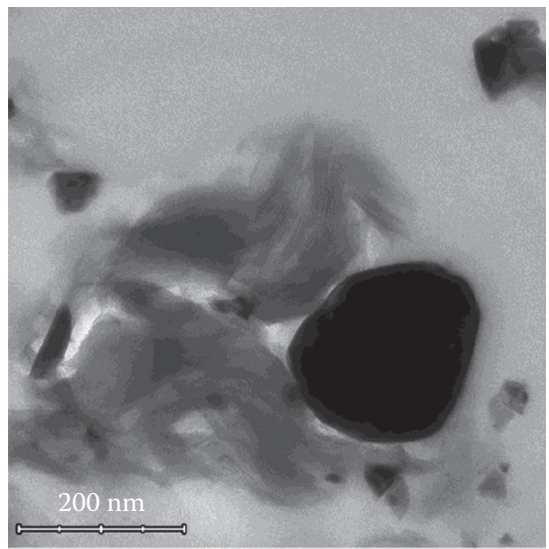

(c)

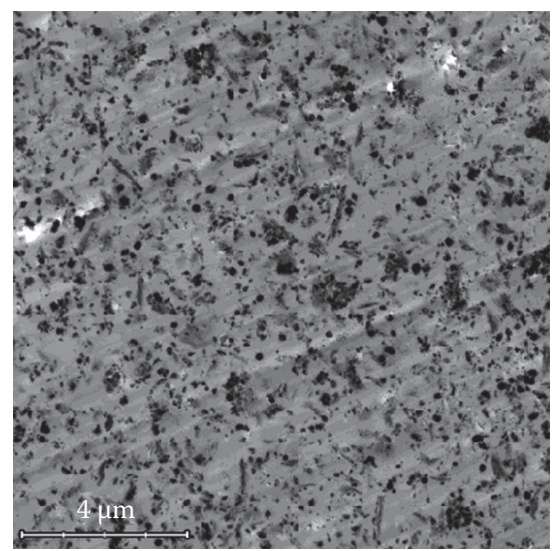

(b)

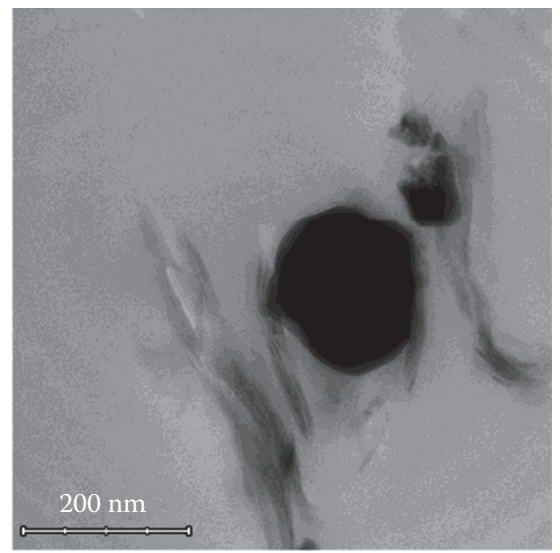

(d)

FIGURE 3.12

TEM images of $\mathrm{BaTiO}_{3} / \mathrm{OMMT}$-based nanocomposites. (a) A phase separated morphology of noncovalently bonded BT and OMMT; (b) a thermodynamically favorable dispersion of covalently bonded $\mathrm{BaTiO}_{3} /$ OMMT fillers; (c and d) higher magnification images of the covalently bonded $\mathrm{BaTiO}_{3} / \mathrm{OMMT}$ nanofillers in the same nanocomposite. 


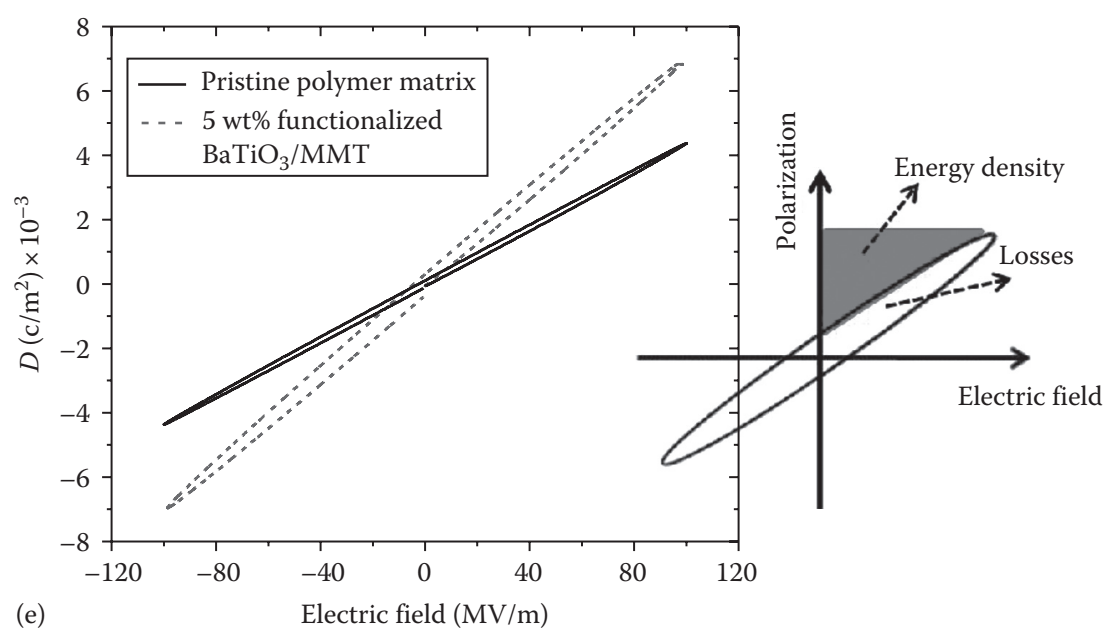

FIGURE 3.12 (continued)

TEM images of $\mathrm{BaTiO}_{3} / \mathrm{OMMT}$-based nanocomposites. (e) a comparison plot of dielectric displacement versus electric field and a schematic illustration of the recoverable energy density. (Reproduced from Polizos, G. et al., J. Appl. Phys., 108, 074117, 2010.)

higher weight fractions in hybrid fillers than the corresponding process in the nonreactive single-filler composites (Figure 3.6), and the activation energy value is $150 \%$ higher than the activation energy for the single BT/ epoxy composites and 20\% higher than the value for the single OMMT/ epoxy composites. Furthermore, the interfacial dynamics of the covalently bonded hybrid composites are several orders of magnitude slower than in the single-phase BT composites. The slower dynamics as well as the increase in the barriers associated to the interfacial relaxations manifest a significant improvement in the strength of the interfaces.

Similar dependencies were observed in the activation energy for the dc conductivity at temperatures above $T_{g}$. A comparison plot between the hybrid filler and the single OMMT composites is shown in Figure 3.14. The hybrid filler composites are characterized by higher activation energy values for all weight fractions; and interestingly, the conduction process in the 5 and $10 \mathrm{wt} \%$ composites is associated with energy barriers higher even than those in the pristine epoxy. When the particle content is lower than the percolation threshold, the cross-linked filler configuration reinforces the interfacial strength and results in marked improvements. In good agreement with the thermoplastic-based composites in Section 3.1, the interfacess and the particle agglomeration morphology dominate the conduction process. Tailoring the nanoscale properties is an effective route for the design and fabrication of composites with optimized performance. In this section, the nanoscale synergistic effects of the slower interfacial dynamics and the respective higher activation energy barriers restricted the mobility of 


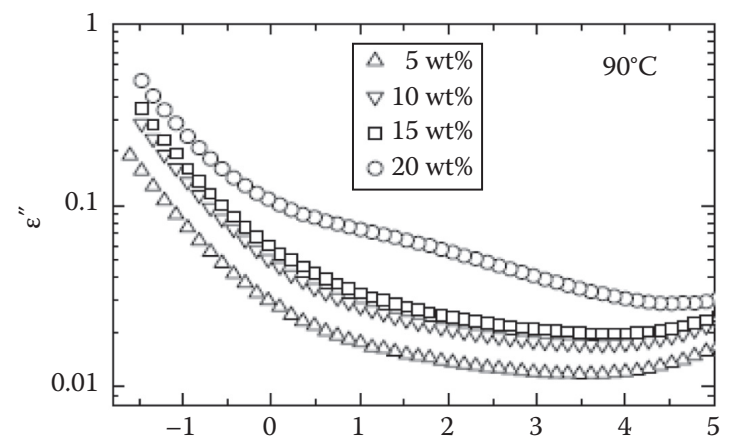

(a)

$\log$ (frequency, $\mathrm{Hz}$ )

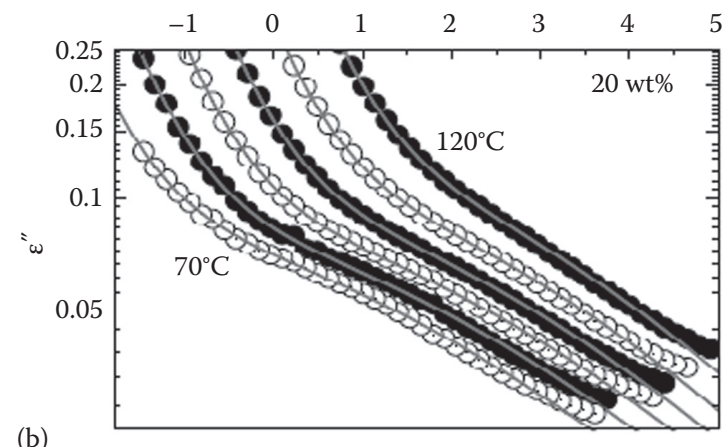

(b)

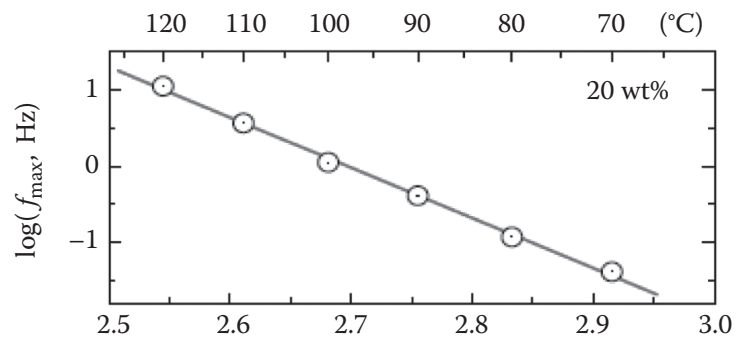

(c)

$1000 / T\left(\mathrm{~K}^{-1}\right)$

FIGURE 3.13

(a) Dielectric loss curves for the hybrid (covalently bonded) composites at different weight fractions for a representative temperature below $T_{g}$. A relaxation process is shaped when the particle content is $20 \mathrm{wt} \%$. (b) This process is due to the formation of a polymer phase loosely bound to the particle surface and is analyzed in the entire temperature range. The lines are the best fits of Equation 3.9 and a conductivity contribution to the experimental data. (c) The corresponding Arrhenius plot obtained from the loss peak maximum frequency. The line is a linear fit of Equation 3.24 to the data. (Reproduced from Polizos, G. et al., J. Appl. Phys., 108, 074117, 2010.) 


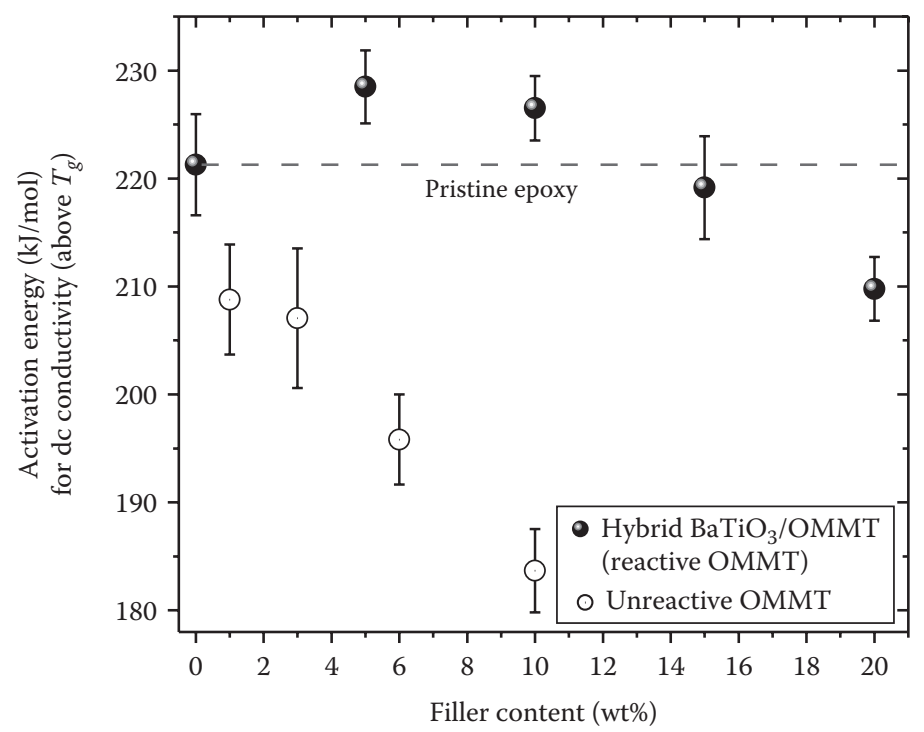

FIGURE 3.14

Activation energies of the conductivity mechanism for the hybrid (covalently bonded) and single filler composites at weight fractions indicated on the plot. (Reproduced from Polizos, G. et al., J. Appl. Phys., 108, 074117, 2010.)

the hybrid composites and resulted to better dielectric breakdown probability distributions [57] that can be utilized in energy storage and electrical insulation applications.

\subsection{Hierarchical Structures}

\subsubsection{Thermoset-Based Nanocomposites}

The hierarchical dispersion of nanoparticulates is an effective method to combine nanometer-scale filler dispersion with long-range ordered structures and optimize certain properties in a desired direction. Herein, we present anisotropic composites with controlled particle distribution and correlate their ordered structure with the dielectric properties [59,60]. A thermoset and a thermoplastic matrix were studied. For the thermoset matrix, a cross-linkable silicone elastomer (polydimethylsiloxane) was used and BT nanoparticles were dielectrophoretically assembled [61-65] by applying high-amplitude ac electric field to the polymer/particle suspension during the curing process. This method utilizes induced polarization effects that arise from the contrast between the high permittivity of the particles and the 
low permittivity of the elastomeric matrix. The time average dielectrophoretic force exerted on the particles is $[66,67]$

$$
\left\langle\vec{F}_{D E P}(t)\right\rangle=2 \pi \varepsilon_{m}^{\prime} R^{3} \operatorname{Re}\left[K^{*}(\omega)\right] \vec{\nabla} E_{r m s}^{2}
$$

where

$\varepsilon_{m}^{\prime}$ denotes the medium's (silicone elastomer) real part of the permittivity function

$R$ is the particle's radius

$E_{r m s}$ is the rms (root mean square) amplitude of the applied field

$K^{*}(\omega)$ is the complex Clausius-Mossotti function that is expressed as

$$
K^{*}(\omega)=\frac{\varepsilon_{p}^{*}-\varepsilon_{m}^{*}}{\varepsilon_{p}^{*}+2 \varepsilon_{m}^{*}}
$$

For both, the particle and the medium $(i=p, m)$, the complex permittivity functions are interpreted in the general form, including also the direct current (dc) conductivity, $\sigma_{d c}$ contribution: $\varepsilon_{i}^{*}(\omega)=\varepsilon_{i}^{\prime}(\omega)-\varepsilon_{i}^{\prime \prime}(\omega)-i \omega^{-1} \sigma_{i, d c}$. Depending on the dielectric functions $\varepsilon_{p}^{*}(\omega)$ and $\varepsilon_{m}^{*}(\omega)$, the frequency of the applied field can be tuned to maximize the $K^{*}(\omega)$ function and therefore the dielectrophoretic force. The resultant column-like aligned structures are shown in Figure 3.15. We follow the sample notation introduced by Newnham [68].

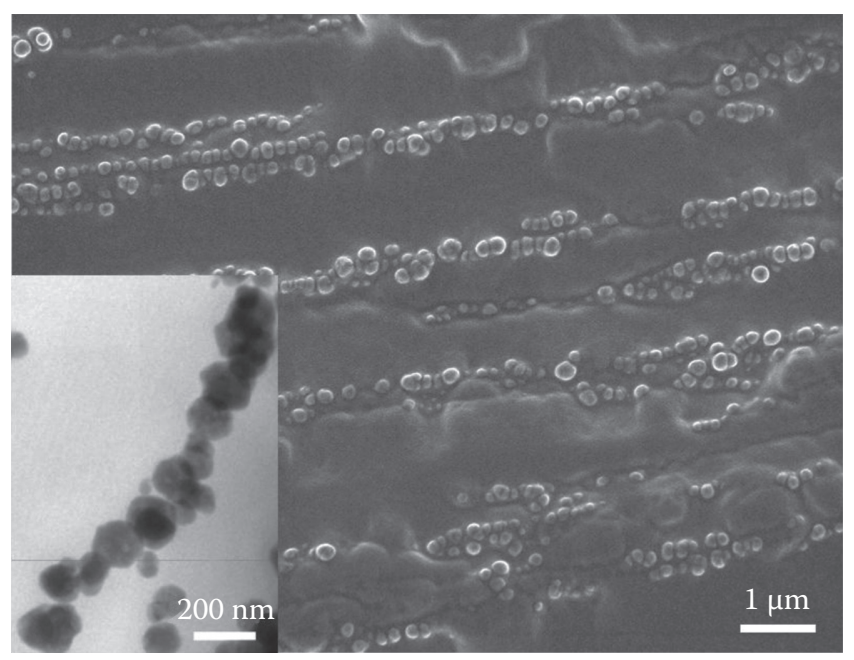

\section{FIGURE 3.15}

SEM and TEM (inset) images of aligned $\mathrm{BaTiO}_{3}(10 \mathrm{vol} \%)$ in silicone elastomer matrix (polydimethylsiloxane). The applied electric field was $1.6 \mathrm{kV} / \mathrm{mm}$ at $100 \mathrm{~Hz}$. (Reproduced from Tomer, V. et al., J. Appl. Phys., 103, 034115, 2008.) 
Specifically, 0-3 denotes a composite with randomly dispersed fillers and 1-3 a composite with fillers aligned in one dimension. 1-3 composites with fillers aligned in the direction of the measuring field (z-axis) are noted as $z$-aligned composite while $x-y$ aligned composites refer to fillers aligned perpendicular to the measuring field.

All composites exhibit higher permittivity than the unfilled polymer matrix. Permittivity values were found to depend on the BT concentration as well as on the spatial distribution of the fillers. A representative plot for the $22.5 \mathrm{vol} \%$ composites is shown in Figure 3.16a. The $z$-aligned (parallel) composite has the highest values over the entire frequency range. At small weight fractions, there is no notable change in the values of the composites; however, at higher fractions, the anisotropy difference is evident (Figure $3.16 \mathrm{~b}$ ) and the $z$-aligned $25 \mathrm{vol} \%$ composite is characterized by approximately four times higher permittivity than the unfilled polymer.

Theoretical models can predict the effective permittivity of a dielectric mixture [69,70]. An example is shown in Figure 3.16c for the 0-3 (randomly dispersed) composites. Among several models, the best fit to the experimental data was obtained by the model of Wakino et al. [71]. This model considers the influence of dielectric and/or infringing of the electric flux due to discontinuity at the boundary of constituent phases and is given by the expression

$$
\varepsilon_{r}=\exp \left[\frac{\ln \left\{V_{c} \varepsilon_{c}^{\left(V_{c}-V_{0}\right)}+\left(1-V_{c}\right) \varepsilon_{p}^{\left(V_{c}-V_{0}\right)}\right\}}{V_{c}-V_{0}}\right]
$$

where

$V_{c}$ is the particle volume fraction

$\varepsilon_{c}$ and $\varepsilon_{p}$ are the particle and polymer permittivities, respectively

$V_{0}$ is the critical volume fraction at which the dispersed phase becomes continuous

In addition to conventional models, the effective permittivity may also be expressed in terms of the spectral density representation by taking into account depolarization factors related to the topology of the interfaces [72]. The hierarchical structure of inorganic fillers with high permittivity and the integration of their interfaces with the polymer matrix is another route for the design of nanocomposites with predicted dielectric properties.

\subsubsection{Thermoplastic-Based Nanocomposites}

Layered silicates modified with dimethyl-dioctadecyl-ammonium were dispersed in maleic anhydride functionalized polyethylene (PE), and nanocomposite films were extruded using a commercial blown-film line [60,73]. 


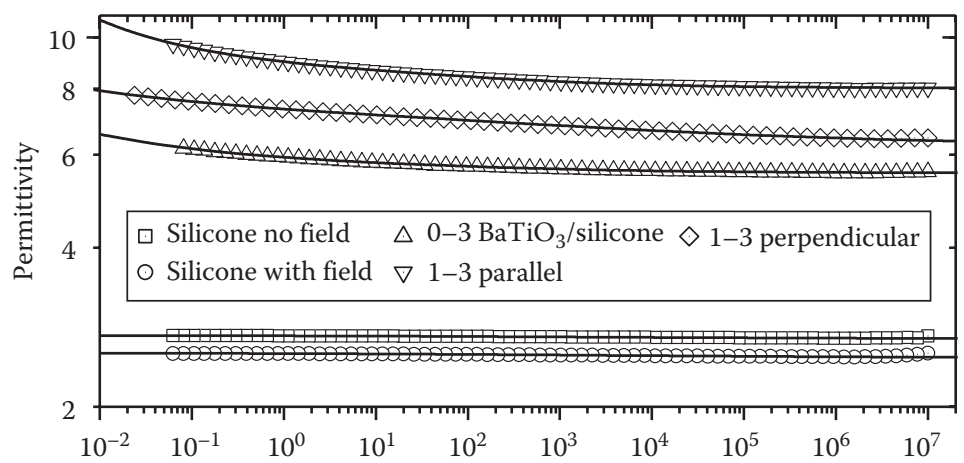

(a)

Frequency $(\mathrm{Hz})$

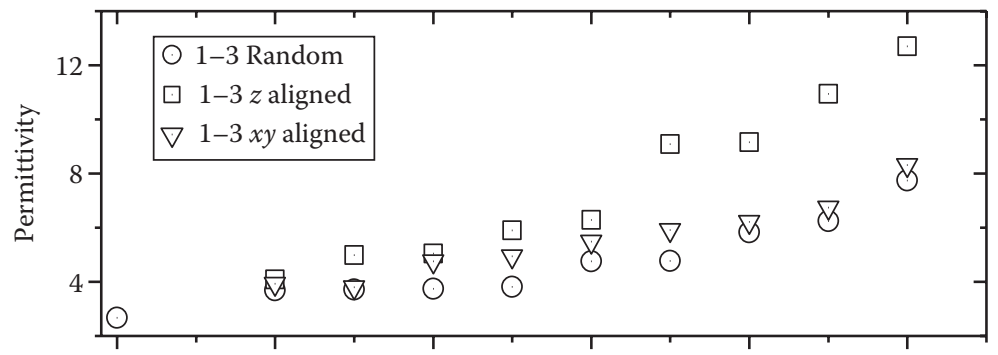

(b)

Ceramic volume fraction (\%)

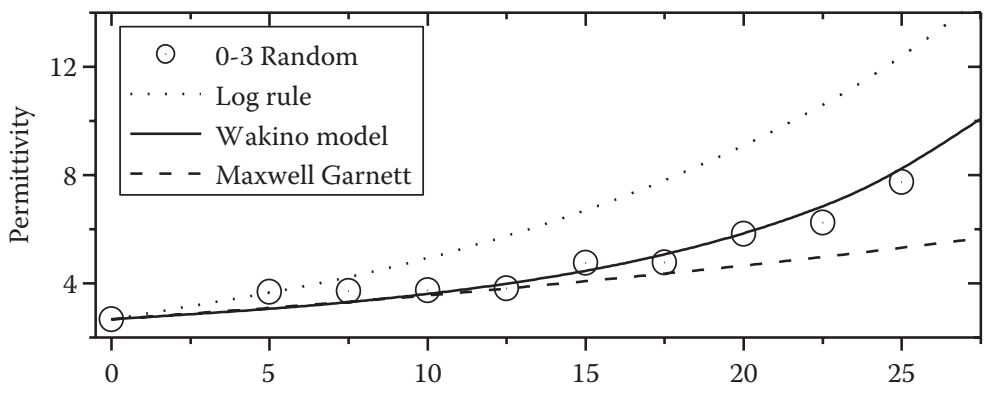

(c)

Ceramic volume fraction (\%)

\section{FIGURE 3.16}

(a) Real part of permittivity at room temperature for the $22.5 \mathrm{vol} \%$ composites and the polydimethylsiloxane matrix (exposed and not exposed to the electric field used for the particle alignment). The lines are the best fits of Equation 3.8 to the data. (b) Permittivity values for the randomly dispersed and structured composites at several weight fractions in $\mathrm{BaTiO}_{3}$. (c) Effective permittivity fitting curves for the randomly dispersed composites according to different mixing rules. (Reproduced from Tomer, V. et al., J. Appl. Phys., 103, 034115, 2008.)

The stress-induced filler orientation was measured by wide-angle x-ray diffraction. The structure of the composites, the diffracted patterns, and the azimuthal profiles are shown in Figure 3.17. The radial intensities $I(\phi)$ were used to determine the order parameter in terms of the Hermans orientation function [74], 

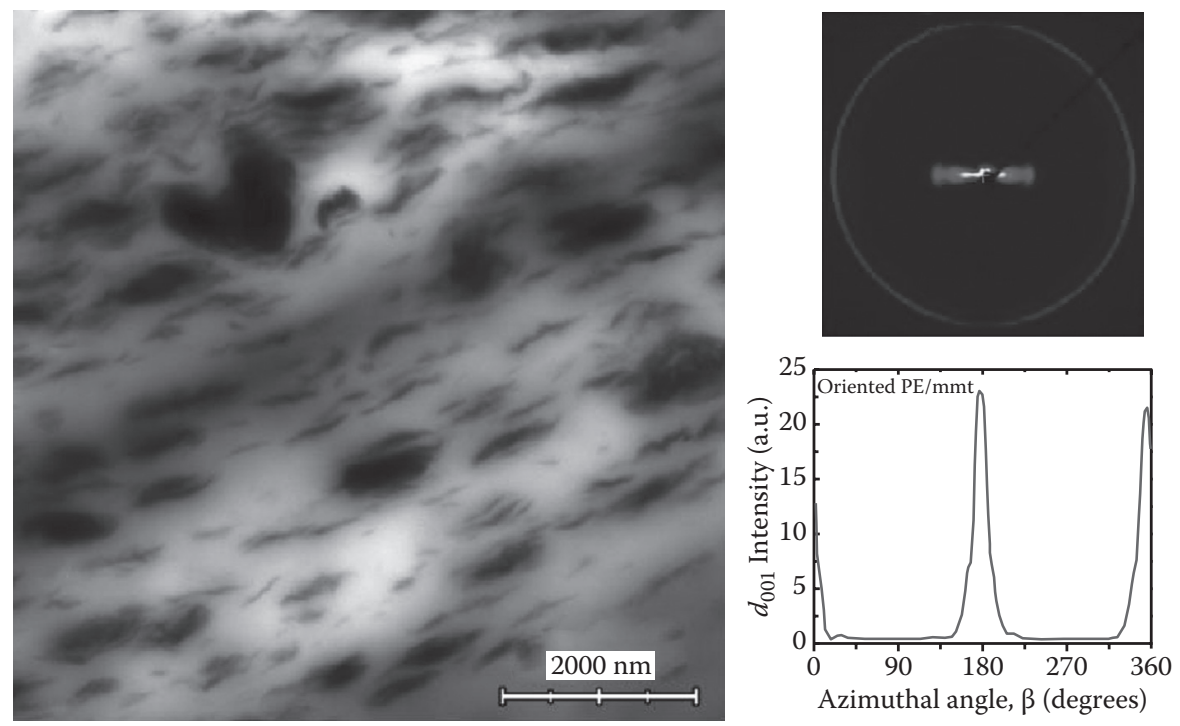

FIGURE 3.17

TEM image of blown-molded polyethylene composites filled with organically modified silicates. The diffracted patterns and the azimuthal profiles are shown on the right plots. The Hermans orientation factor is 0.8. (Reproduced from Tomer, V. et al., J. Appl. Phys., 109, 074113, 2011.)

$$
S_{d}=\frac{3\left\langle\cos ^{2} \phi\right\rangle-1}{2}
$$

where

$$
\left\langle\cos ^{2} \phi\right\rangle=\frac{\int_{0}^{\pi / 2} I(\phi) \cos ^{2} \phi \sin \phi d \phi}{\int_{0}^{\pi / 2} I(\phi) \sin \phi d \phi}
$$

The $S_{d}$ values for perfectly aligned and randomly dispersed particles are 1 and 0 , respectively; and the calculated $S_{d}$ factor for the oriented fillers in Figure 3.17 was found to be 0.8 , manifesting a high-degree orientation at the direction of blow-molding.

In good agreement with the impedance analysis in Section 3.1, dielectric spectroscopy in the permittivity formalism probes two relaxation mechanisms that are present in the composites only and shown in Figure 3.18. They involve interactions between space-charge and polar segments at the 

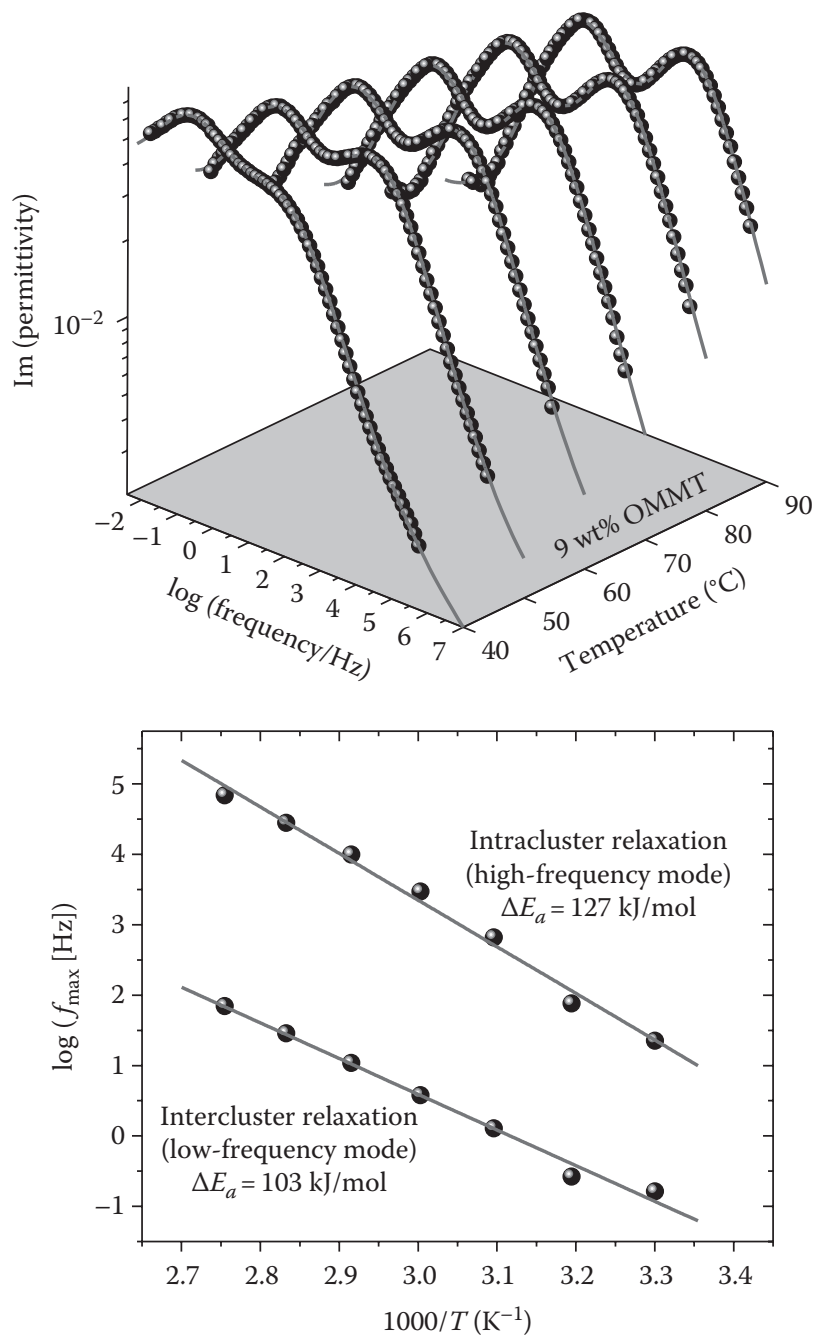

FIGURE 3.18

Dielectric relaxations for the aligned polymer nanocomposite in Figure 3.17. The lines are the best fits of a superposition of two Havriliak-Negami expressions (Equation 3.9) to the data. The corresponding Arrhenius plots and activation energies are shown on the bottom plot. (Reproduced from Tomer, V. et al., J. Appl. Phys. 109, 074113, 2011.)

silicate interfaces (intracluster relaxation at high frequencies) as well as interactions between space-charge and polar maleic anhydride segments in the bulk polyethylene phase amid neighboring silicate clusters (intercluster relaxation at low frequencies). The energy barriers associated with these relaxations are quantified by the respective activation energies. Their values, presented in Figure 3.18, are typical for ion conduction mechanisms and the activation energy for the intracluster relaxation was found to be 


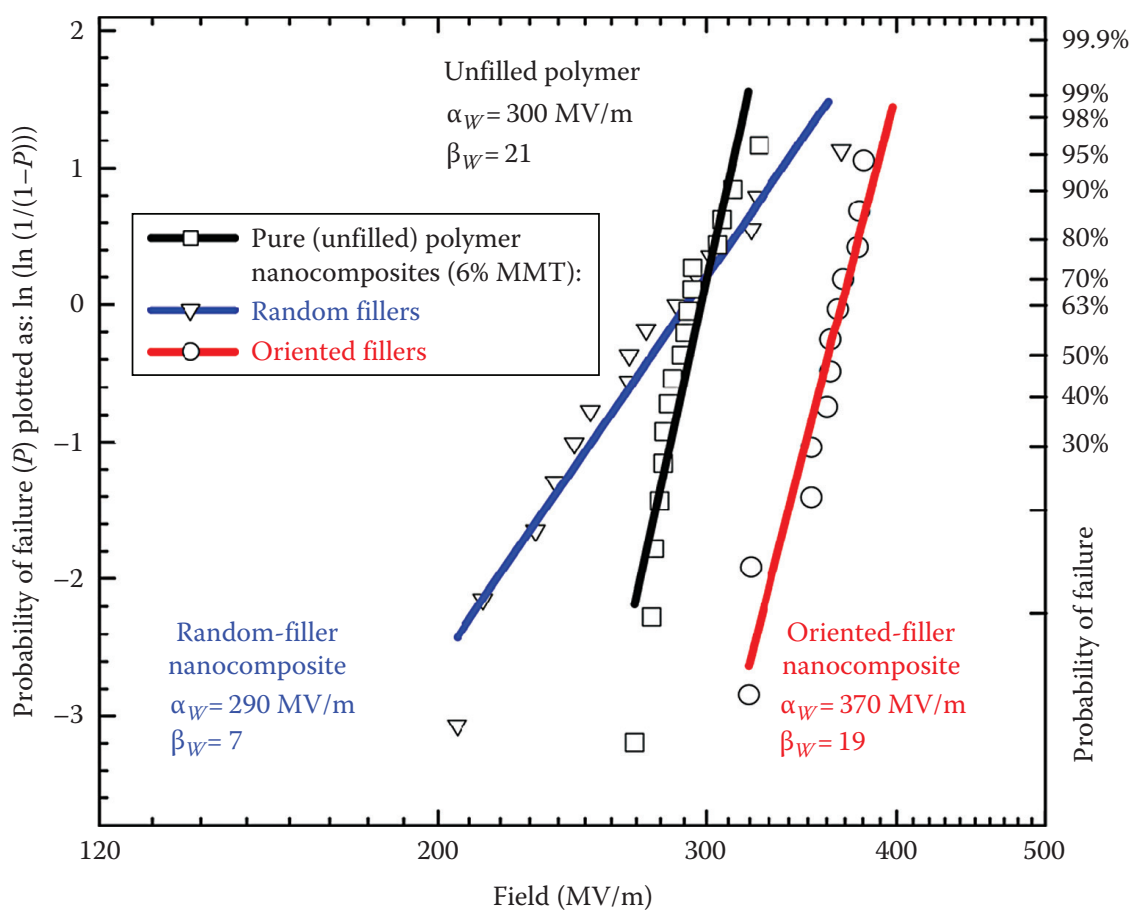

FIGURE 3.19

(See color insert.) Dielectric breakdown failure distributions for the unfilled polymer matrix and the nanocomposites based on randomly and aligned layered silicates. The lines are linear fits to the Weibull cumulative probability and the characteristic parameters are indicated on the plot. (Reproduced from Tomer, V. et al., J. Appl. Phys., 109, 074113, 2011.)

significantly higher due to the strong electrostatic interactions with the silicate surface.

The aligned configuration of the silicate particles has shown outstanding improvement in the dielectric breakdown failure distributions of the nanocomposites, presented in Figure 3.19.

The characteristic breakdown strength was found to qualitatively change behavior with the particle orientation and was markedly increased for the oriented structures, where random filler structures showed the typical onset of failure decreasing to much lower fields than for the matrix. This improvement was the result of synergistic effects related to the ordered structure of high aspect ratio silicates (that force more tortuous paths for the propagation of charges), and to the modification of the interfaces (that create traps for the space-charge, increasing the energy barriers for their diffusion and, therefore, minimizing the bulk charge accumulation). 


\subsection{Summary}

The introduction of inorganic nanoparticles into a polymer matrix unavoidably defines interfaces, which often determine the properties and performance of the composite. In this chapter, we presented aspects of our research efforts over the past years on identifying critical structural parameters that mitigate some of the detrimental effects associated with these interfaces and can expand the operating and application windows of the polymers. The size of the particles comparable to polymer length scales, the particle functionality, and the highly ordered self-assembled configurations are key parameters in designing multifunctional composites with markedly enhanced capabilities. The phenomenal growth of research and commercialization of hybrid nanostructured materials necessitates the development and utilization of techniques that accurately probe properties at the nanoscale. DRS combined with proper analysis tools are a versatile method for dynamically measuring nanoscale properties, separating synergistic contributions, and predicting the macroscopic behavior of composites. This chapter focused on such spectroscopic studies for composites with potential dielectrical applications and with promise to store electrostatic energy. Across a broad range of polymer matrices, the controlled distribution of the fillers and the tailored interfaces according to dielectric measurements resulted to improvements that are outstanding in the field of nanodielectrics and provide the grounds for further development and optimization.

\section{Acknowledgments}

The work presented in this chapter was supported by the Office of Naval Research (Grant No. MURI-00014-05-1-0541), the National Science Foundation (Grant No. DMR-0602877), and the U.S. Department of EnergyOffice of Electricity Delivery and Energy Reliability, Advanced Cables and Conductors Program for Electric Power Systems (under contract DE-AC0500OR22725 with Oak Ridge National Laboratory, managed and operated by UT-Battelle, LLC).

Part of this work was performed at Oak Ridge National Laboratory's Center for Nanophase Materials Sciences, sponsored by the Scientific User Facilities Division, Office of Basic Energy Sciences, and U.S. Department of Energy, The National Science foundation I/UCRC Center for Dielectrics Studies. 


\section{References}

1. F. Kremer, A. Schönhals (eds.), Broadband Dielectric Spectroscopy, Springer-Verlag, Berlin, Germany (2002).

2. N.G. McCrum, B.E. Read, G. Williams, Anelastic and Dielectric Effects in Polymeric Solids, Wiley, London, U.K. (1967).

3. G. Williams, D.K. Thomas, Novocontrol Appl. Note Dielectr. 3, 1-28 (1998).

4. C.J.F. Boettcher, P. Bordewijk, Theory of Electric Polarization, 2nd edn., vol. 2, Elsevier, Amsterdam, the Netherlands, p. 72 (1978).

5. S. Havriliak, S. Negami, J. Polym. Sci., Polym. Symp. 14, 89 (1966).

6. F. Alvarez, A. Alegria, J. Colmenero, Phys. Rev. B 44, 7306 (1991).

7. A. Boersma, J. van Turnhout, M. Wübbenhorst, Macromolecules 31, 7453 (1998).

8. K.S. Cole, R.H. Cole, J. Chem. Phys. 9, 341 (1941).

9. M. Wübbenhorst, J. van Turnhout, J. Non-Cryst. Solids 305, 40 (2002).

10. F.S. Howell, C.T. Moynihan, P.B. Macedo, Bull. Chem. Soc. Jpn. 57, 652 (1984).

11. K. Pathmanathan, G.P. Johari, J. Chem. Phys. 95, 5990 (1991).

12. P.B. Macedo, C.T. Moynihan, R. Bose, Phys. Chem. Glasses 13, 171 (1972).

13. C.T. Moynihan, L.P. Boesch, N.L. Laberge, Phys. Chem. Glasses 14, 122 (1973).

14. J.H. Ambrus, C.T. Moynihan, P.B. Macedo, J. Phys. Chem. 76, 3287 (1972).

15. G.M. Tsangaris, G.C. Psarras, N. Kouloumbi, J. Mater. Sci. 33, 2027 (1998).

16. G.C. Psarras, E. Manolakaki, G.M. Tsangaris, Compos. Part A: Appl. Sci. Manuf. 33, 375 (2002).

17. J.D. Jacobs, H. Koerner, H. Heinz, B.L. Farmer, P. Mirau, P.H. Garrett, R.A. Vaia, J. Phys. Chem. B 110, 20143-20157 (2006).

18. J.K. Nelson, Y. Hu, J. Phys. D: Appl. Phys. 38, 213-222 (2005).

19. Y. Cao, P.C. Irwin, K. Younsi, IEEE Trans. Dielectr. Electr. Insul. 11, 797-807 (2004).

20. E. Tuncer, I. Sauers, D.R. James, A.R. Ellis, M.P. Paranthaman, T. Aytug, S. Sathyamurthy, K.L. More, J. Li, A. Goyal, Nanotechnology 18, 025703 (2007).

21. M. Volel, M. Armand, W. Gorecki, M.L. Saboungi, Chem. Mater. 17, 2028-2033 (2005).

22. J.-D. Jeon, M.-J. Kim, S.-Y. Kwak, J. Power Sources 162, 1304-1311 (2006).

23. G. Polizos, E. Tuncer, A.L. Agapov, D. Stevens, A.P. Sokolov, M.K. Kidder, J.D. Jacobs, H. Koerner, R.A. Vaia, K.L. More, I. Sauers, Polymer 53, 595-603 (2012).

24. P. Pissis, G. Polizos, Molecular dynamics and ionic conductivity studies in polyurethane thermoplastic elastomers, Chapter 14, in Handbook of Condensation Thermoplastic Elastomers, S. Fakirov (ed.), Wiley-VCH, Weinheim, Germany (2005).

25. P. Pissis, L. Apekis, C. Christodoulides, M. Niaounakis, A. Kyritsis, J. Nedbal, J. Polym. Sci., Polym. Phys. 34, 1529-1539 (1996).

26. E. Malmstrom, A. Hult, U.W. Gedde, F. Liu, R.H. Boyd, Polymer 38, 4873-4879 (1997).

27. P.W. Zhu, S. Zheng, G. Simon, Macromol. Chem. Phys. 202, 3008-3017 (2001).

28. M.S. Kim, P.K. Sekhar, S. Bhansali, J.P. Harmon, J. Nanosci. Nanotechnol. 9, 5776-5784 (2009).

29. G. Turky, S.S. Shaaban, A, Schönhals, J. Appl. Polym. Sci. 113, 2477-2484 (2009). 
30. R. Böhmer, K.L. Ngai, C.A. Angell, D.J. Plazek, J. Chem. Phys. 99, 4201-4209 (1993).

31. R. Richert, C.A. Angell, J. Chem. Phys. 108, 9016-9026 (1998).

32. R. Böhmer, C.A. Angell, Disorder Effects on Relaxational Processes, R. Richert and A. Blumen (eds.), Springer, Berlin, Germany (1994).

33. E. Rössler, A.P. Sokolov, Chem. Geol. 128, 143-153 (1996).

34. H. Couderc, A. Saiter, J. Grenet, J.M. Saiter, G. Boiteux, E. Nikaj, I. Stevenson, N. D'Souza, Polym. Eng. Sci. 49, 836-843 (2009).

35. H.C. Wong, A. Sanz, J.F. Douglas, J.T. Cabral, J. Mol. Liq. 153, $79-87$ (2010).

36. J. Jancar, J.F. Douglas, F.W. Starr, S.K. Kumar, P. Cassagnau, A.J. Lesser, S.S. Sternstein, M.J. Buehler, Polymer 51, 3321-3343 (2010).

37. Y. Ding, S. Pawlus, A.P. Sokolov, J.F. Douglas, A. Karim, C.L. Soles, Macromolecules 42, 3201-3206 (2009).

38. G. Polizos, V.V. Shilov, P. Pissis, J. Non-Cryst. Solids 305, 212-217 (2002).

39. C.A. Solunov, Eur. Polym. J. 35, 1543-1556 (1999).

40. A. Saiter, J.M. Saiter, J. Grenet, Eur. Polym. J. 42, 213-219 (2006).

41. V. Tomer, G. Polizos, E. Manias, C.A. Randall, J. Appl. Phys. 108, 074116 (2010).

42. J. Mijovic, H. Zhang, Macromolecules 36, 1279 (2003).

43. J.C. Maxwell, A Treatise on Electricity and Magnetism, Clarendon Press, Oxford, U.K. 1, p. 452 (1892).

44. R.W. Sillars, J. Inst. Electron. Eng. 80, 378 (1937).

45. K.W. Wagner, Electr. Eng. (Arch. Elektrotech.) 2, 371 (1914).

46. K. Funke, B. Roling, M. Lange, Solid State Ionics 105, 195 (1998).

47. B. Roling, C. Martiny, K. Funke, J. Non-Cryst. Solids 249, 201 (1999).

48. E. Tuncer, I. Sauers, D.R. James, A.R. Ellis, M.P. Paranthaman, A. Goyal, K.L. More, Nanotechnology 18, 325704 (2007).

49. D.H. Lee, J.G. Park, K.J. Choi, H.J. Choi, D.W. Kim, Eur. J. Inorg. Chem. 6, 878-882 (2008).

50. C.D. Lokhande, E.H. Lee, K.D. Jung, O.S. Joo, J. Mater. Sci. 39, 2915-2918 (2004).

51. S. Cassaignon, M. Koelsch, J.P. Jolivet, J. Phys. Chem. Solids 68, 695-700 (2007).

52. F. Pedraza, A. Vazquez, J. Phys. Chem. Solids 60, 445-448 (1999).

53. M. Kiyama, T. Akita, Y. Tsutsumi, T. Takada, Chem. Lett. 1, 21-24 (1972).

54. G. Polizos, E. Tuncer, I. Sauers, K.L. More, Appl. Phys. Lett. 96, 152903 (2010).

55. W. Weibull, J. Appl. Mech. 18, 293-297 (1951).

56. S.M. Rowland, R.M. Hill, L.A. Dissado, J. Phys. C: Solid State Phys. 19, 6263-6285 (1986).

57. G. Polizos, V. Tomer, E. Manias, C.A. Randall, J. Appl. Phys. 108, 074117 (2010).

58. Manias, G. Hadziioannou, G. ten Brinke, J. Chem. Phys. 101, 1721-1724 (1994).

59. V. Tomer, C.A. Randall, G. Polizos, J. Kostelnick, E. Manias, J. Appl. Phys. 103, 034115 (2008).

60. V. Tomer, G. Polizos, C.A. Randall, E. Manias, J. Appl. Phys. 109, 074113 (2011).

61. C.P. Bowen, T.R. Shrout, R.E. Newnham, C.A. Randall, J. Int. Mater. Syst. Struct. 6, 159 (1995).

62. C.P. Bowen, R.E. Newnham, C.A. Randall, J. Mater. Res. 13, 205 (1998).

63. J.E. Martin, C.P. Tigges, R.A. Anderson, Phys. Rev. B 60, 7127 (1999).

64. C.A. Randall, D.V. Miller, J.H. Adair, A.S. Bhalla, J. Mater. Res. 8, 899 (1993).

65. S.A. Wilson, G.M. Maistros, R.W. Whatmore J. Phys. D: Appl. Phys. 38, 175 (2005).

66. H.A. Pohl, Dielectrophoresis, Cambridge University Press, Cambridge, U.K. (1978). 
67. T.B. Jones, Electromechanics of Particles, Cambridge University Press, Cambridge, U.K. (1995).

68. R.E. Newnham, Ferroelectrics 68, 1 (1986).

69. P.S. Neelakanta, Handbook of Electromagnetic Materials: Monolithic and Composite Versions and Their Applications, CRC Press, Boca Raton, FL (1995).

70. A. Sihvola, Electromagnetic Mixing Formulas and Applications, The Institution of Electrical Engineers, London, U.K. (1999).

71. K. Wakino, T. Okada, N. Yoshida, K. Tomono, J. Am. Ceram. Soc. 76, 2588 (1993).

72. E. Tuncer, Appl. Phys. A 107, 575-582 (2012).

73. J. Zhang, E. Manias, G. Polizos, J.-Y. Huh, A. Ophir, P. Songtipya, M.M. JimenezGasco, J. Adhes. Sci. Technol. 23, 709 (2009).

74. H. Koerner, Y. Luo, X. Li, C. Cohen, R. Hedden, C. Ober, Macromolecules 36, 1975 (2003). 


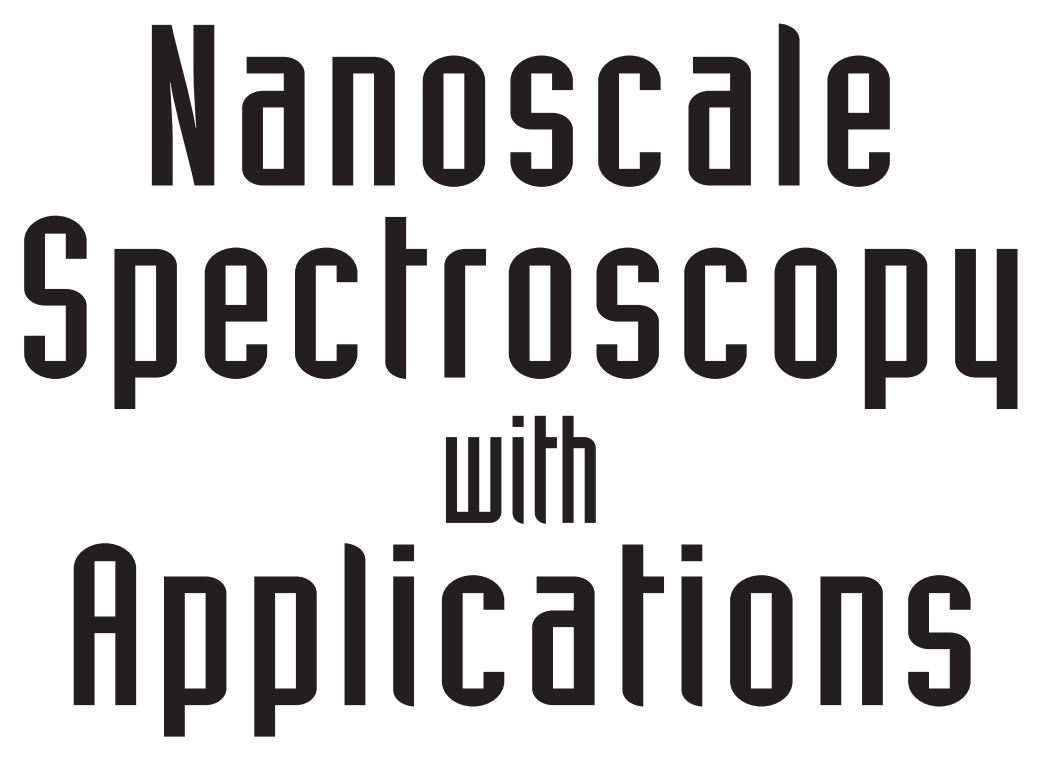

\section{Edited by \\ Sarhan M. Musa}

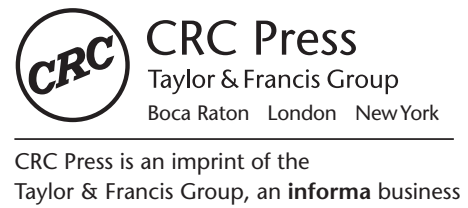


MATLAB $^{\circ}$ is a trademark of The MathWorks, Inc. and is used with permission. The MathWorks does not warrant the accuracy of the text or exercises in this book. This book's use or discussion of MATLAB ${ }^{\circ}$ software or related products does not constitute endorsement or sponsorship by The MathWorks of a particular pedagogical approach or particular use of the MATLAB ${ }^{\circ}$ software.

CRC Press

Taylor \& Francis Group

6000 Broken Sound Parkway NW, Suite 300

Boca Raton, FL 33487-2742

(C) 2014 by Taylor \& Francis Group, LLC

CRC Press is an imprint of Taylor \& Francis Group, an Informa business

No claim to original U.S. Government works

Version Date: 20130923

International Standard Book Number-13: 978-1-4665-6854-9 (eBook - PDF)

This book contains information obtained from authentic and highly regarded sources. Reasonable efforts have been made to publish reliable data and information, but the author and publisher cannot assume responsibility for the validity of all materials or the consequences of their use. The authors and publishers have attempted to trace the copyright holders of all material reproduced in this publication and apologize to copyright holders if permission to publish in this form has not been obtained. If any copyright material has not been acknowledged please write and let us know so we may rectify in any future reprint.

Except as permitted under U.S. Copyright Law, no part of this book may be reprinted, reproduced, transmitted, or utilized in any form by any electronic, mechanical, or other means, now known or hereafter invented, including photocopying, microfilming, and recording, or in any information storage or retrieval system, without written permission from the publishers.

For permission to photocopy or use material electronically from this work, please access www.copyright.com (http://www.copyright.com/) or contact the Copyright Clearance Center, Inc. (CCC), 222 Rosewood Drive, Danvers, MA 01923, 978-750-8400. CCC is a not-for-profit organization that provides licenses and registration for a variety of users. For organizations that have been granted a photocopy license by the CCC, a separate system of payment has been arranged.

Trademark Notice: Product or corporate names may be trademarks or registered trademarks, and are used only for identification and explanation without intent to infringe.

Visit the Taylor \& Francis Web site at

http://www.taylorandfrancis.com

and the CRC Press Web site at

http://www.crcpress.com 